EUROPEAN LABORATORY FOR PARTICLE PHYSICS

CERN-PPE/97-086

17 July 1997

\title{
Measurement of the quark to photon fragmentation function through the inclusive production of prompt photons in hadronic $\mathrm{Z}^{0}$ decays
}

\author{
The OPAL Collaboration
}

\begin{abstract}
The inclusive production of prompt photons with energy above $10 \mathrm{GeV}$ is measured using the OPAL detector in hadronic $\mathrm{Z}^{0}$ decays at LEP. In contrast to previous measurements, the prompt photons were not required to be isolated. The production rate and energy spectrum are found to be in agreement with QCD predictions for the quark-to-photon fragmentation function.
\end{abstract}

(To be submitted to Zeitschrift für Physik C) 


\section{The OPAL Collaboration}

K. Ackerstaff ${ }^{8}$, G. Alexander ${ }^{23}$, J. Allison ${ }^{16}$, N. Altekamp ${ }^{5}$, K.J. Anderson ${ }^{9}$, S. Anderson ${ }^{12}$, S. Arcelli ${ }^{2}$, S. Asai ${ }^{24}$, D. Axen ${ }^{29}$, G. Azuelos ${ }^{18, a}$, A.H. Ball $^{17}$, E. Barberio ${ }^{8}$, T. Barillari² ${ }^{2}$ R.J. Barlow ${ }^{16}$, R. Bartoldus ${ }^{3}$, J.R. Batley ${ }^{5}$, S. Baumann ${ }^{3}$, J. Bechtluft ${ }^{14}$, C. Beeston ${ }^{16}$, T. Behnke ${ }^{8}$, A.N. Bell ${ }^{1}$, K.W. Bell ${ }^{20}$, G. Bella ${ }^{23}$, S. Bentvelsen ${ }^{8}$, S. Bethke ${ }^{14}$, O. Biebel ${ }^{14}$, A. Biguzzi ${ }^{5}$, S.D. Bird ${ }^{16}$, V. Blobel ${ }^{27}$, I.J. Bloodworth ${ }^{1}$, J.E. Bloomer ${ }^{1}$, M. Bobinski ${ }^{10}$, P. Bock ${ }^{11}$, D. Bonacorsi ${ }^{2}$, M. Boutemeur ${ }^{34}$, B.T. Bouwens ${ }^{12}$, S. Braibant ${ }^{12}$, L. Brigliadori ${ }^{2}$, R.M. Brown ${ }^{20}$, H.J. Burckhart ${ }^{8}$, C. Burgard ${ }^{8}$, R. Bürgin ${ }^{10}$, P. Capiluppi ${ }^{2}$, R.K. Carnegie ${ }^{6}$, A.A. Carter $^{13}$, J.R. Carter ${ }^{5}$, C.Y. Chang ${ }^{17}$, D.G. Charlton ${ }^{1, b}$, D. Chrisman ${ }^{4}$, P.E.L. Clarke ${ }^{15}$,

I. Cohen ${ }^{23}$, J.E. Conboy ${ }^{15}$, O.C. Cooke ${ }^{8}$, M. Cuffiani ${ }^{2}$, S. Dado ${ }^{22}$, C. Dallapiccola ${ }^{17}$,

G.M. Dallavalle ${ }^{2}$, R. Davies ${ }^{30}$, S. De Jong ${ }^{12}$, L.A. del Pozo ${ }^{4}$, K. Desch ${ }^{3}$, B. Dienes ${ }^{33, d}$, M.S. Dixit ${ }^{7}$, E. do Couto e Silva ${ }^{12}$, M. Doucet ${ }^{18}$, E. Duchovni ${ }^{26}$, G. Duckeck ${ }^{34}$, I.P. Duerdoth ${ }^{16}$, D. Eatough ${ }^{16}$, J.E.G. Edwards ${ }^{16}$, P.G. Estabrooks ${ }^{6}$, H.G. Evans ${ }^{9}$, M. Evans ${ }^{13}$, F. Fabbri ${ }^{2}$, M. Fanti ${ }^{2}$, A.A.Faust ${ }^{30}$, F. Fiedler ${ }^{27}$, M. Fierro ${ }^{2}$, H.M. Fischer ${ }^{3}$, I. Fleck ${ }^{8}$, R. Folman ${ }^{26}$, D.G. Fong ${ }^{17}$, M. Foucher ${ }^{17}$, A. Fürtjes ${ }^{8}$, D.I. Futyan $^{16}$, P. Gagnon ${ }^{7}$, J.W. Gary ${ }^{4}$, J. Gascon ${ }^{18}$, S.M. Gascon-Shotkin ${ }^{17}$, N.I. Geddes ${ }^{20}$, C. Geich-Gimbel ${ }^{3}$, T. Geralis ${ }^{20}$, G. Giacomelli ${ }^{2}$, P. Giacomelli ${ }^{4}$, R. Giacomelli ${ }^{2}$, V. Gibson ${ }^{5}$, W.R. Gibson ${ }^{13}$, D.M. Gingrich ${ }^{30, a}$, D. Glenzinski ${ }^{9}$, J. Goldberg ${ }^{22}$, M.J. Goodrick ${ }^{5}$, W. Gorn ${ }^{4}$, C. Grandi ${ }^{2}$, E. Gross ${ }^{26}$, J. Grunhaus ${ }^{23}$, M. Gruwé ${ }^{8}$,

C. Hajdu ${ }^{32}$, G.G.Hanson ${ }^{12}$, M. Hansroul ${ }^{8}$, M. Hapke ${ }^{13}$, C.K. Hargrove ${ }^{7}$, P.A. Hart ${ }^{9}$,

C. Hartmann ${ }^{3}$, M. Hauschild ${ }^{8}$, C.M.Hawkes ${ }^{5}$, R. Hawkings ${ }^{27}$, R.J.Hemingway ${ }^{6}$,

M. Herndon ${ }^{17}$, G. Herten ${ }^{10}$, R.D. Heuer ${ }^{8}$, M.D. Hildreth ${ }^{8}$, J.C. Hill ${ }^{5}$, S.J. Hillier ${ }^{1}$,

P.R. Hobson ${ }^{25}$, R.J.Homer ${ }^{1}$, A.K. Honma ${ }^{28, a}$, D. Horváth ${ }^{32, c}$, K.R. Hossain ${ }^{30}$, R. Howard ${ }^{29}$, P. Hüntemeyer ${ }^{27}$, D.E. Hutchcroft ${ }^{5}$, P. Igo-Kemenes ${ }^{11}$, D.C. Imrie ${ }^{25}$, M.R. Ingram ${ }^{16}$, K. Ishii ${ }^{24}$, A. Jawahery ${ }^{17}$, P.W. Jeffreys ${ }^{20}$, H. Jeremie ${ }^{18}$, M. Jimack ${ }^{1}$, A. Joly ${ }^{18}$, C.R. Jones ${ }^{5}$, G. Jones ${ }^{16}$, M. Jones ${ }^{6}$, U. Jost ${ }^{11}$, P. Jovanovic ${ }^{1}$, T.R. Junk ${ }^{8}$, D. Karlen ${ }^{6}$, V. Kartvelishvili ${ }^{16}$, K. Kawagoe ${ }^{24}$, T. Kawamoto ${ }^{24}$, P.I. Kayal ${ }^{30}$, R.K. Keeler ${ }^{28}$, R.G. Kellogg ${ }^{17}$, B.W. Kennedy ${ }^{20}$, J. Kirk ${ }^{29}$, A. Klier ${ }^{26}$, S. Kluth ${ }^{8}$, T. Kobayashi ${ }^{24}$, M. Kobel ${ }^{10}$, D.S. Koetke ${ }^{6}$, T.P. Kokott ${ }^{3}$, M. Kolrep ${ }^{10}$, S. Komamiya ${ }^{24}$, T. Kress ${ }^{11}$, P. Krieger ${ }^{6}$, J. von Krogh ${ }^{11}$, P. Kyberd ${ }^{13}$, G.D. Lafferty ${ }^{16}$, R. Lahmann ${ }^{17}$, W.P. Lai ${ }^{19}$, D. Lanske ${ }^{14}$, J. Lauber ${ }^{15}$, S.R. Lautenschlager ${ }^{31}$, J.G. Layter ${ }^{4}$, D. Lazic ${ }^{22}$, A.M. Lee ${ }^{31}$, E. Lefebvre ${ }^{18}$, D. Lellouch ${ }^{26}$, J. Letts ${ }^{12}$, L. Levinson ${ }^{26}$, S.L. Lloyd ${ }^{13}$, F.K. Loebinger ${ }^{16}$, G.D. Long ${ }^{28}$, M.J. Losty ${ }^{7}$, J. Ludwig ${ }^{10}$, A. Macchiolo ${ }^{2}$, A. Macpherson ${ }^{30}$, M. Mannelli ${ }^{8}$, S. Marcellini' ${ }^{2}$, C. Markus ${ }^{3}$, A.J. Martin ${ }^{13}$, J.P. Martin ${ }^{18}$, G. Martinez ${ }^{17}$, T. Mashimo ${ }^{24}$, P. Mättig ${ }^{3}$, W.J. McDonald ${ }^{30}$, J. McKenna ${ }^{29}$, E.A. Mckigney ${ }^{15}$,

T.J. McMahon ${ }^{1}$, R.A. McPherson ${ }^{8}$, F.Meijers ${ }^{8}$, S. Menke ${ }^{3}$, F.S. Merritt ${ }^{9}$, H. Mes ${ }^{7}$, J. Meyer ${ }^{27}$, A. Michelini' ${ }^{2}$, G. Mikenberg ${ }^{26}$, D.J.Miller ${ }^{15}$, A. Mincer ${ }^{22, e}$, R. Mir ${ }^{26}$, W. Mohr ${ }^{10}$,

A. Montanari ${ }^{2}$, T. Mori ${ }^{24}$, M. Morii ${ }^{24}$, U. Müller ${ }^{3}$, S. Mihara ${ }^{24}$, K. Nagai ${ }^{26}$, I. Nakamura ${ }^{24}$, H.A. Neal ${ }^{8}$, B. Nellen ${ }^{3}$, R. Nisius ${ }^{8}$, S.W. O’Neale ${ }^{1}$, F.G. Oakham ${ }^{7}$, F. Odorici ${ }^{2}$, H.O. Ogren ${ }^{12}$, A. $\mathrm{Oh}^{27}$, N.J. Oldershaw ${ }^{16}$, M.J. Oreglia ${ }^{9}$, S. Orito ${ }^{24}$, J. Pálinkás ${ }^{33, d}$, G. Pásztor ${ }^{32}$, J.R. Pater ${ }^{16}$, G.N. Patrick ${ }^{20}$, J. Patt ${ }^{10}$, M.J. Pearce ${ }^{1}$, R. Perez-Ochoa8, S. Petzold ${ }^{27}$, P. Pfeifenschneider ${ }^{14}$, J.E. Pilcher ${ }^{9}$, J.Pinfold ${ }^{30}$, D.E. Plane ${ }^{8}$, P. Poffenberger ${ }^{28}$, B. Poli ${ }^{2}$, A. Posthaus ${ }^{3}$, D.L. Rees ${ }^{1}$, D. Rigby ${ }^{1}$, S. Robertson ${ }^{28}$, S.A. Robins ${ }^{22}$, N. Rodning ${ }^{30}$,

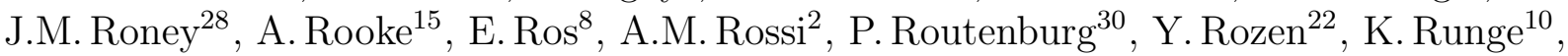


O. Runolfsson ${ }^{8}$, U. Ruppel ${ }^{14}$, D.R. Rust ${ }^{12}$, R. Rylko ${ }^{25}$, K. Sachs ${ }^{10}$, T. Saeki ${ }^{24}$, E.K.G. Sarkisyan ${ }^{23}$, C. Sbarra ${ }^{29}$, A.D. Schaile ${ }^{34}$, O. Schaile ${ }^{34}$, F. Scharf ${ }^{3}$, P. Scharff-Hansen ${ }^{8}$, P. Schenk ${ }^{34}$, J. Schieck ${ }^{11}$, P. Schleper ${ }^{11}$, B. Schmitt ${ }^{8}$, S. Schmitt ${ }^{11}$, A. Schöning ${ }^{8}$, M. Schröder ${ }^{8}$, H.C. Schultz-Coulon ${ }^{10}$, M. Schumacher ${ }^{3}$, C. Schwick ${ }^{8}$, W.G. Scott ${ }^{20}$, T.G. Shears ${ }^{16}$, B.C. Shen ${ }^{4}$, C.H. Shepherd-Themistocleous ${ }^{8}$, P. Sherwood ${ }^{15}$, G.P. Siroli ${ }^{2}$, A. Sittler ${ }^{27}$, A. Skillman ${ }^{15}$, A. Skuja ${ }^{17}$, A.M. Smith ${ }^{8}$, G.A.Snow ${ }^{17}$, R. Sobie ${ }^{28}$, S. Söldner-Rembold ${ }^{10}$, R.W.Springer ${ }^{30}$, M. Sproston ${ }^{20}$, K. Stephens ${ }^{16}$, J. Steuerer ${ }^{27}$, B. Stockhausen ${ }^{3}$, K. Stoll ${ }^{10}$, D. Strom ${ }^{19}$, P. Szymanski ${ }^{20}$, R. Tafirout ${ }^{18}$, S.D. Talbot ${ }^{1}$, S. Tanaka ${ }^{24}$, P. Taras ${ }^{18}$, S. Tarem ${ }^{22}$,

R. Teuscher ${ }^{8}$, M. Thiergen ${ }^{10}$, M.A. Thomson ${ }^{8}$, E. von Törne ${ }^{3}$, S. Towers ${ }^{6}$, I. Trigger ${ }^{18}$, Z. Trócsányi ${ }^{33}$, E. Tsur ${ }^{23}$, A.S. Turcot ${ }^{9}$, M.F. Turner-Watson ${ }^{8}$, P. Utzat ${ }^{11}$, R. Van Kooten ${ }^{12}$, M. Verzocchi ${ }^{10}$, P. Vikas ${ }^{18}$, E.H. Vokurka ${ }^{16}$, H. Voss ${ }^{3}$, F. Wäckerle ${ }^{10}$, A. Wagner ${ }^{27}$, C.P. Ward ${ }^{5}$, D.R. Ward ${ }^{5}$, P.M. Watkins ${ }^{1}$, A.T. Watson ${ }^{1}$, N.K. Watson ${ }^{1}$, P.S. Wells ${ }^{8}$, N. Wermes ${ }^{3}$, J.S. White ${ }^{28}$, B. Wilkens ${ }^{10}$, G.W.Wilson ${ }^{27}$, J.A. Wilson ${ }^{1}$, G. Wolf ${ }^{26}$, T.R. Wyatt ${ }^{16}$, S. Yamashita ${ }^{24}$, G. Yekutieli ${ }^{26}$, V. Zacek ${ }^{18}$, D. Zer-Zion ${ }^{8}$

${ }^{1}$ School of Physics and Space Research, University of Birmingham, Birmingham B15 2TT, UK

${ }^{2}$ Dipartimento di Fisica dell' Università di Bologna and INFN, I-40126 Bologna, Italy

${ }^{3}$ Physikalisches Institut, Universität Bonn, D-53115 Bonn, Germany

${ }^{4}$ Department of Physics, University of California, Riverside CA 92521, USA

${ }^{5}$ Cavendish Laboratory, Cambridge CB3 0HE, UK

${ }^{6}$ Ottawa-Carleton Institute for Physics, Department of Physics, Carleton University, Ottawa, Ontario K1S 5B6, Canada

${ }^{7}$ Centre for Research in Particle Physics, Carleton University, Ottawa, Ontario K1S 5B6, Canada

${ }^{8}$ CERN, European Organisation for Particle Physics, CH-1211 Geneva 23, Switzerland

${ }^{9}$ Enrico Fermi Institute and Department of Physics, University of Chicago, Chicago IL 60637, USA

${ }^{10}$ Fakultät für Physik, Albert Ludwigs Universität, D-79104 Freiburg, Germany

${ }^{11}$ Physikalisches Institut, Universität Heidelberg, D-69120 Heidelberg, Germany

${ }^{12}$ Indiana University, Department of Physics, Swain Hall West 117, Bloomington IN 47405, USA

${ }^{13}$ Queen Mary and Westfield College, University of London, London E1 4NS, UK

${ }^{14}$ Technische Hochschule Aachen, III Physikalisches Institut, Sommerfeldstrasse 26-28, D52056 Aachen, Germany

${ }^{15}$ University College London, London WC1E 6BT, UK

${ }^{16}$ Department of Physics, Schuster Laboratory, The University, Manchester M13 9PL, UK

${ }^{17}$ Department of Physics, University of Maryland, College Park, MD 20742, USA

${ }^{18}$ Laboratoire de Physique Nucléaire, Université de Montréal, Montréal, Quebec H3C 3J7, Canada

${ }^{19}$ University of Oregon, Department of Physics, Eugene OR 97403, USA

${ }^{20}$ Rutherford Appleton Laboratory, Chilton, Didcot, Oxfordshire OX11 0QX, UK

${ }^{22}$ Department of Physics, Technion-Israel Institute of Technology, Haifa 32000, Israel 
${ }^{23}$ Department of Physics and Astronomy, Tel Aviv University, Tel Aviv 69978, Israel

${ }^{24}$ International Centre for Elementary Particle Physics and Department of Physics, University of Tokyo, Tokyo 113, and Kobe University, Kobe 657, Japan

${ }^{25}$ Brunel University, Uxbridge, Middlesex UB8 3PH, UK

${ }^{26}$ Particle Physics Department, Weizmann Institute of Science, Rehovot 76100, Israel

${ }^{27}$ Universität Hamburg/DESY, II Institut für Experimental Physik, Notkestrasse 85, D-22607 Hamburg, Germany

${ }^{28}$ University of Victoria, Department of Physics, P O Box 3055, Victoria BC V8W 3P6, Canada

${ }^{29}$ University of British Columbia, Department of Physics, Vancouver BC V6T 1Z1, Canada

${ }^{30}$ University of Alberta, Department of Physics, Edmonton AB T6G 2J1, Canada

${ }^{31}$ Duke University, Dept of Physics, Durham, NC 27708-0305, USA

${ }^{32}$ Research Institute for Particle and Nuclear Physics, H-1525 Budapest, P O Box 49, Hungary

${ }^{33}$ Institute of Nuclear Research, H-4001 Debrecen, P O Box 51, Hungary

${ }^{34}$ Ludwigs-Maximilians-Universität München, Sektion Physik, Am Coulombwall 1, D-85748 Garching, Germany

${ }^{a}$ and at TRIUMF, Vancouver, Canada V6T $2 \mathrm{~A} 3$

${ }^{b}$ and Royal Society University Research Fellow

${ }^{c}$ and Institute of Nuclear Research, Debrecen, Hungary

${ }^{d}$ and Department of Experimental Physics, Lajos Kossuth University, Debrecen, Hungary

$e^{e}$ and Department of Physics, New York University, NY 1003, USA 


\section{Introduction}

We gain a greater understanding of the properties of the elementary building blocks of matter and their interactions by studying the properties of hadrons, leptons and photons produced as a result of a primary interaction. The properties of hadrons and charged leptons produced in $\mathrm{e}^{+} \mathrm{e}^{-}$collisions have been studied in great detail at different centre-of-mass energies. In the case of photons radiated off quarks, prompt photons, in hadronic $\mathrm{e}^{+} \mathrm{e}^{-}$collisions, much less information is available due to the difficulty of separating these photons from those produced in the decays of other particles [1]-[5]. Both the shape and normalisation of the inclusive prompt photon energy spectrum in $\mathrm{e}^{+} \mathrm{e}^{-}$collisions are predicted, through the calculation of the quark-to-photon fragmentation function, by leading-order perturbative QCD [6, 7]. This asymptotic prediction has been parametrised in [8]. Non-perturbative effects can be included in the calculation through the vector-meson dominance ansatz as in [9, 10], where boundary terms missing in 8] were also accounted for. The higher-order terms were calculated, and seen to be small at the energies close to the $\mathrm{Z}^{0}$ peak. Direct experimental study of these predictions is important in providing insight into the non-perturbative and higher order effects in the radiation of photons from quarks. This will also make theoretical predictions of photon production in other processes, such as those occuring at pp and $\mathrm{p} \overline{\mathrm{p}}$ colliders, more reliable, thus improving sensitivity to possible new phenomena.

At LEP the first measurement of prompt photon production in hadronic $\mathrm{Z}^{0}$ decays was made by the OPAL Collaboration [11] for photons isolated from other particles in the event, as suggested in [12]. The production of isolated prompt photons was studied in great detail by all LEP experiments [13]-16]. Following the suggestion of [17], the ALEPH Collaboration extracted the quark-to-photon fragmentation function from the study of non-isolated photons in jets containing a photon carrying more than $70 \%$ of the jet energy 18 .

Here we present a measurement of the inclusive prompt photon energy spectrum in hadronic $\mathrm{Z}^{0}$ decays at LEP. This method of studying the quark-to-photon fragmentation function was suggested in [10, 19]. To separate prompt photons from the photons from decays of other particles we use the following method. We selected clusters in the electromagnetic calorimeter not associated with charged tracks. A set of cuts were applied to reduce the background in the sample. The distribution of a variable characterising the transverse shape of the clusters in data was then fitted with a linear combination of the distributions for photons and for background to determine the fraction of prompt photons in the selected sample. The result was then corrected for the selection efficiencies, detector effects and initial state radiation. In the following sections we describe the OPAL detector, the event and electromagnetic cluster selection (sec. 3) and the determination of the number of photons in the selected sample (sec. 母). The efficiency and acceptance corrections are described (sec. 5) followed by the study of systematic effects (sec. 6). Finally the measured prompt photon energy spectrum is presented and discussed (sec. 7). 


\section{The OPAL detector}

The OPAL detector operates at the LEP $\mathrm{e}^{+} \mathrm{e}^{-}$collider at CERN. A detailed description of the detector can be found in [20]. For this study, the most important components of OPAL were the central detector and the barrel electromagnetic calorimeter with its presampling detector. The central detector, measuring the momenta of charged particles, consists of a system of cylindrical tracking chambers surrounded by a solenoidal coil which produces a uniform axial magnetic field of $0.435 \mathrm{~T}$ along the beam axis is almost $100 \%$ within the polar angle range $|\cos \theta|<0.95$.

The electromagnetic calorimeters completely cover the azimuthal range for polar angles satisfying $|\cos \theta|<0.98$ providing excellent hermeticity. The barrel electromagnetic calorimeter covers the polar angle range $|\cos \theta|<0.82$. It consists of 9440 lead glass blocks, each 24.6 radiation lengths deep, almost pointing towards the interaction region. Each block subtends an angular region of approximately $40 \times 40 \mathrm{mrad}^{2}$. Half of the block width corresponds to 1.9 Moliére radii. Deposits of energy in adjacent blocks are grouped together to form clusters of electromagnetic energy. The intrinsic energy resolution of $\sigma_{\mathrm{E}} / \mathrm{E}=0.2 \% \oplus 6.3 \% / \sqrt{\mathrm{E}}$ is substantially degraded (by a factor $\simeq 2$ ) due to the presence of two radiation lengths of material in front of the lead glass. For the intermediate region, $0.72<|\cos \theta|<0.82$, the amount of material increases up to eight radiation lengths causing further degradation in the energy resolution. The two endcap calorimeters, each made of 1132 lead glass blocks, 22 radiation lengths deep, cover the region of $0.81<|\cos \theta|<0.98$. In this study the measurement of inclusive photon production is restricted to the barrel part of the detector. Most of the electromagnetic showers start before the calorimeter and their position at the entrance of the calorimeter is measured by a barrel electromagnetic presampler made of limited streamer mode chambers. The presampler covers the polar angle range $|\cos \theta|<0.81$ and its angular resolution for photons is approximately $2 \mathrm{mrad}$.

\section{The selection of events and electromagnetic clusters}

Our study was based on a sample of 2.5 million hadronic $\mathrm{Z}^{0}$ decays selected as described in 21] from the data accumulated with the OPAL detector at LEP in 1992, 1993 and 1994 at an $\mathrm{e}^{+} \mathrm{e}^{-}$centre-of-mass energy of $91.2 \mathrm{GeV}$. We did not use the off-peak data to avoid additional complications in dealing with data collected at different $\mathrm{e}^{+} \mathrm{e}^{-}$centre-of-mass energies. We required that the central detector and the calorimeters were fully operational. Temporary, local inefficiencies in the presampler chambers were monitored and taken into account.

To study the properties of the background we used Monte Carlo events produced with the parton shower generators JETSET 7.4 [22] (3.9 million events) and HERWIG 5.8 [23] (1.1 million events) with generator parameters given in [24]. We also used samples of events with only single photon or a $\pi^{0}$ meson present in the detector. The Monte Carlo (MC) samples

\footnotetext{
${ }^{1}$ In the OPAL coordinate system the $x$ axis points towards the centre of the LEP ring, the $y$ axis points upwards and the $z$ axis points in the direction of the electron beam. The polar angle $\theta$ and the azimuthal angle $\phi$ are defined with respect to the $z$ and $x$-axes, respectively, while $r$ is the distance from the $z$-axis.
} 
were passed through the full simulation of the OPAL detector [25] and subjected to the same reconstruction and analysis procedure as the data.

The difficulty in the measurement of prompt photon production lay in the separation of the signal from background. The QCD shower models predicted a signal-to-background ratio of approximately $1 / 200$. Background clusters, with no charged track associated with them, are dominated by photons from decays of hadrons, particularly $\pi^{0} \rightarrow \gamma \gamma(\simeq 57 \%)$ and $\eta \rightarrow \gamma \gamma(\simeq 10 \%)$. The other sources of background, like the interaction of neutral hadrons such as $\mathrm{K}_{\mathrm{L}}^{0}$ 's or neutrons in the material of the calorimeter contribute at the level of few per cent each. More than one particle can also contribute to a cluster. The transverse profile of the calorimeter cluster can be used to differentiate between clusters coming from different sources. The hardest to remove are clusters produced by $\pi^{0} \rightarrow \gamma \gamma$ and $\eta \rightarrow \gamma \gamma$ meson decays because they can be very similar, especially for higher cluster energies, to those produced by one photon. An irreducible, but very well predicted, background comes from the initial state radiation (ISR) photons radiated by the beam particles before they interacted.

The tracks and calorimeter clusters were selected as described in [21. In addition we required a cluster energy to be larger than $10 \mathrm{GeV}$ and cluster polar angle such that $|\cos \theta|<$ 0.72. We then applied three cuts motivated by studies with simulated events.

Cut 1. There was required to be no charged track associated with the cluster. Tracks were extrapolated to the calorimeter surface. A track was associated with a calorimeter cluster if it extrapolates to the calorimeter within $24 \mathrm{mrad}$ (approximately half of a lead glass block width) of the centre of gravity of a cluster. In Figure 1a and 1 b, the normalised distributions of the angle between the calorimeter cluster and the nearest track are shown for background clusters and prompt photons in the JETSET model, for small and large cluster energies. For the higher energy clusters a contribution from electrons and positrons from conversions of prompt photons in the beam pipe and central detector is seen for small angles $\delta$. For lower energy clusters this effect is diluted both by the greater separation between the extrapolation of the track to the calorimeter surface and the centre of gravity of the calorimeter cluster, and by the presence of other tracks in the proximity of the prompt photon.

Cut 2. We required the presence of a presampler cluster within $24 \mathrm{mrad}$ of the centre of gravity of the calorimeter cluster. The differences in azimuthal $|\Delta \phi|$ and polar $|\Delta \Theta|$ angles between the positions of the calorimeter cluster and the presampler cluster were required to satisfy $\Delta=\min (|\Delta \phi|,|\Delta \Theta|)<\Delta_{\text {cut }}$. The distributions of $\Delta$ for single, isolated photons and $\pi^{0}$ 's in the detector as well as all background clusters in JETSET events are shown in Fig. 2 $2 \mathrm{a}$ and $2 \mathrm{~b}$. The value of $\Delta$ tended to be larger for clusters produced by two overlapping photons, e.g. from a $\pi^{0} \rightarrow \gamma \gamma$ than for clusters produced by a single photon, because the presampler measures the cluster position at an early stage of development of the electromagnetic shower.

Cut 3. The transverse profile of the cluster in the calorimeter was required to be compatible with that produced by an isolated photon. It was first assumed that the cluster was produced by a photon. The impact point of the photon was varied until the best description of the 
observed lateral shower profile by a reference profile was found. The reference profile was obtained by the parametrisation of the results of $\mathrm{MC}$ simulation of the isolated photon in the detector. The fast algorithm described in section 4.1 of [26] was used. The resulting variable, $S$, is proportional to the $\chi^{2}$ for matching the measured and predicted energy sharing between the calorimeter blocks. The distributions of $S$ for single photons, single $\pi^{0}$ 's and background clusters in JETSET events are shown in Fig. 20 and 2dd.

The energy dependent values of $\Delta_{\text {cut }}$ and $S_{\text {cut }}$ were chosen to optimise the separation of signal from background and are shown in Tab. 11.

In total 23106 clusters passed the selection procedure. The signal-to-background ratio, estimated from simulation, was improved from 1/200 to $1 / 130$ after cut 1 , then to $1 / 50$ after cut 2 and finally to $1 / 6$ after cut 3 with respectively $92 \%, 51 \%$ and $42 \%$ of the signal retained. According to the simulation the background clusters passing the selection were produced mostly by $\pi^{0} \rightarrow \gamma \gamma(\simeq 79 \%)$ and $\eta \rightarrow \gamma \gamma(\simeq 11 \%)$ decays.

The cuts lead to a strong reduction of the background while prompt photons were much less affected. The fraction of photons rejected by the cuts can be corrected for and its knowledge will affect the systematic uncertainty of the measurement as detailed in Sections 5 and 6 . The efficiency of cuts 2 and 3 for photon clusters well separated from other particles in the event was determined directly from the data using a sample of photons in radiative lepton pair events $\mathrm{e}^{+} \mathrm{e}^{-} \rightarrow \ell^{+} \ell^{-} \gamma(\ell=\mathrm{e}, \mu)$. In addition, in hadronic events, further losses of prompt photons occur when other particles hit the calorimeter close to the photon. This can result in a cluster being associated to a track or being sufficiently distorted to fail the selection criteria. A small fraction $(\simeq 6 \%)$ of prompt photons also converted in the beam pipe or central detector. The correction for these effects was estimated using Monte Carlo as detailed in sec. 5. In Figure 11 we compare the data and $\mathrm{MC}$ distributions for the angle $\delta$ between the cluster and track closest to it. Differences between data and Monte Carlo are concentrated in the region of small angles, below our cut value of $24 \mathrm{mrad}$, a region containing only a small fraction of prompt photons. In Figure 3 we present data and $\mathrm{MC}$ distributions for the variables $S$ and $\Delta$ used in cuts 2 and 3 . We compare distributions for photons from radiative lepton events with MC for single, isolated photons (a and c). We also show distributions for data and MC clusters from the $\tau^{+} \tau^{-}$events $\left(\tau^{ \pm} \rightarrow \rho^{ \pm} \nu_{\tau}\right.$ and $\rho^{ \pm} \rightarrow \pi^{ \pm} \pi^{0}$, section 4.2 of [26]) (b and d). The

differences between data and MC are small and concentrated mostly in the region below our cut values, so they will cause only small systematic effects.

\section{The determination of the number of photons in the selected sample}

We determined the fraction of photons in the sample remaining after cuts 1-3 above using the cluster shape fit variable $C$ used in the previous OPAL studies of photon production [11, 16]. The fit algorithm applied was more sophisticated than that used in the the calculation of the variable $S$. The variable $C$ had a better background rejection power than $S$. Due to 
the similar fit algorithms and shower parametrisations the $C$ and $S$ variables are correlated, although not fully. The definition of $C$ is

$$
C=\frac{1}{N_{b}} \sum_{i} \frac{\left(E_{i}^{\mathrm{pred}}-E_{i}^{\mathrm{obs}}\right)^{2}}{\left(\sigma_{i}^{\mathrm{pred}}\right)^{2}+\left(\sigma_{i}^{\mathrm{obs}}\right)^{2}}
$$

where: $E_{i}^{\text {obs }}$ is the energy observed in calorimeter block number $i ; E_{i}^{\text {pred }}$ is the predicted energy in calorimeter block number $i ; \sigma_{i}^{\text {pred }}$ and $\sigma_{i}^{\text {obs }}$ are the energy dependent errors on $E_{i}^{\text {pred }}$ and $E_{i}^{\text {obs }}$, respectively; and $N_{b}$ is the number of blocks in the cluster. $E_{i}^{\text {pred }}$ was taken from the best fit of the shower profile parametrisation, assuming that the cluster was produced by a isolated photon, to the observed energy sharing between the calorimeter blocks. The reference profiles varied as a function of $\cos \theta$ because of the varying amount of material in front of the calorimeter.

We fitted the distribution of $C$ in the data with a linear combination of MC distributions for photons and background for clusters passing the same selection criteria as the data:

$$
C_{\text {fit }}=f C_{\gamma}+(1-f) C_{\mathrm{bkg}} \text {. }
$$

The fraction $f$ of photons in the selected sample was the fit parameter. For the distribution of the background, $C_{\mathrm{bkg}}$, clusters from JETSET hadronic $\mathrm{Z}^{0}$ events were used, where initial state radiation and prompt photons were removed from the sample. For the distribution of the photons, $C_{\gamma}$, we used a simulated sample of isolated photons. The fit for $f$ to the $C$ variable distribution in the data was performed separately in seven bins of cluster energy as shown in first column of Tables 1 and 2. The $C_{\gamma}$ and $C_{\mathrm{bkg}}$ distributions had only a small dependence on cluster energy within a given bin. A binned maximum likelihood method [27 was used to fit the $C$ variable distribution between 0 and 5 .

Since we used simulated distributions for $C_{\gamma}$ and $C_{\mathrm{bkg}}$ in eq. 2, it is crucial to check that the $C$ variable is well described in the simulation. We show data and MC distributions for photons (from radiative lepton pair events and single, isolated photon MC) in Fig. 3e and clusters from data and MC $\tau$ decays $\left(\tau^{ \pm} \rightarrow \rho^{ \pm} \nu_{\tau}\right.$ and $\rho^{ \pm} \rightarrow \pi^{ \pm} \pi^{0}$, sec. 4.2 in [26]) in Fig. 3f. The simulation describes well the $C$ variable distributions for clusters produced by isolated photons as well as by $\pi^{0}$ 's. In Fig. 5 we show distributions of the $C$ variable for background clusters from different sources in the JETSET simulation. The shapes of the distributions are similar, although $\pi^{0} \rightarrow \gamma \gamma$ and $\eta \rightarrow \gamma \gamma$ decays tend to produce less clusters with higher values of $C$ than other sources of background.

The fitted fraction of photons $f$ is shown in Tab. 2 for different cluster energy ranges. The comparison of the data and fit results is given in Fig. 4 . The contributions from prompt photons and background to the fit are shown. The $\chi^{2}$, taking into account the statistical errors on the $C_{\gamma}$ and $C_{\mathrm{bkg}}$ distributions, were between 16 and 37 for 23 degrees-of-freedom. 


\section{Corrections for efficiency, acceptance and initial state radiation}

The energy spectrum of prompt photons obtained in the previous section was corrected for photons lost in the selection process and outside the geometrical acceptance. To ensure that the energy spectrum of photons was not biased, efficiency corrections were determined separately for each energy bin. Then the contribution due to initial state radiation was subtracted and the corrected photon energy spectrum was normalised to the total number of hadronic events.

We applied corrections for the following effects.

1. Local, temporary inefficiencies in the presampler system. This factor was determined from data to be 1.23 with negligible statistical error.

2. Rejection of real photons by cuts 2 and 3 . A correction was determined using the data sample of photons in radiative lepton pair events.

3. Additional rejection, by the combined effects of cuts 1,2 and 3 , of photons that formed calorimeter clusters with other particles in the event or converted in the beam-pipe or central detector. This correction, called photon environment in Tab. 1, was determined with the JETSET Monte Carlo as the ratio of the combined cut efficiency for prompt photons where no other particle contributed to the calorimeter cluster, to the efficiency for all prompt photons.

4. The contribution of initial state radiation, estimated using the KORALZ program version 4.0, was subtracted.

Values of the energy dependent corrections are shown in Tab. 1. To compare our result with theoretical predictions we applied an additional correction for the rejection of photons by the cut on the polar angle $\theta(|\cos \theta|<0.72)$. The correction was 1.58 . We assumed the leadingorder $1+\cos ^{2} \theta$ dependence of the photon production cross-section, which was consistent with the polar angle distribution of the selected calorimeter clusters. The fully corrected energy spectrum of prompt photons in hadronic $\mathrm{Z}^{0}$ decays is shown in Tab. 2 and in Fig. 6 .

\section{$6 \quad$ Systematic effects}

We checked the dependence of our result on possible deficiencies in the simulation of the detector and on the particle composition of the background. The main sources of the systematic uncertainties were estimated as follows:

1. The sensitivity of the fit result to the quality of the $\mathrm{MC}$ reproduction of the $C$ variable was determined as follows, separately for each cluster energy bin. The $C_{\gamma}$ and $C_{\mathrm{bkg}}$ distributions used in the fit were simultaneously scaled by $(1 \pm \alpha)$, where $\alpha(\simeq 4 \%)$ was 
the error on the mean value of $C$ from the Monte Carlo added in quadrature to that from the data. These modified distributions were then used in the fit. The differences between these results and those obtained with unmodified distributions were assigned as the systematic error. These uncertainties, ranging from 10-23\% and partly correlated between different photon energy points, were the dominant source of systematic error in our measurement.

2. The fractions of photons in the data obtained from the fit using the background spectrum predicted by the HERWIG MC were in agreement with those obtained using the JETSET $\mathrm{MC}$ background. This showed that, within our statistical precision, the result did not depend on the details of the model implementation of the parton shower development and hadronisation processes in the MC generators. The difference between the fraction of prompt photons obtained from the data fitted with the $C_{\mathrm{bkg}}$ predicted by the JETSET and HERWIG was assigned as a systematic error.

3. Although we relied on the data as much as possible in the determination of the efficiency corrections we had to resort to MC to estimate how the efficiencies for photons passing the selection cuts were modified by the presence of other particles in the event (correction 3 in sec. 5). The corrections obtained with JETSET and HERWIG models gave results consistent within statistical errors despite the differences in the modelling of the prompt photon radiation, parton shower and hadronisation for the two models. The difference between corrections obtained with the JETSET and HERWIG models was assigned as a systematic error.

In addition several further checks were performed, none of which produced a statistically significant, at the one standard deviation level, difference from the result of the default procedure and were not included in the systematic error.

- In equation 2 the amount of the background in the sample was the fit parameter. Therefore, the fitted value of $f$ was not, to first order, sensitive to the background flux as incorporated in the $\mathrm{MC}$ generator. In principle, some sensitivity to the relative fluxes of different background sources remained, since it could change the shape of the $C$ variable distribution of the background. This sensitivity was estimated by repeating the fit with the number of clusters in the background produced by $\eta$ mesons, the second largest source of background, adjusted by the uncertainty on the $\eta$ yield of $\pm 20 \%$ [29]. This resulted in $1.5 \%$ change of the prompt photon yield.

- The systematic error from the modelling of the $C$ variable (point 1 ) was consistent with an estimate which assumed a dependence of the factor $\alpha$ on the value of $C$ variable. For $C$ greater than 2 the factor $\alpha$ was put to zero. For lower $C$ values a linear dependence was assumed, such that the mean value of $\alpha$ for $C$ between 0 and 2 was equal to the value of $\alpha$ used in the default procedure.

- The fit of equation 2 was repeated with the distribution of $C$ for prompt photons in JETSET used as $C_{\gamma}$ instead of the default of isolated photons.

- The values of the cuts $\Delta_{\text {cut }}, S_{\text {cut }}$ and for the association cut were changed by $\pm 20 \%$. 
- The fit result (section 4) did not depend on the bin size of the fitted distribution (changed by a factor of 2) or the upper fit boundary (moved between 5 and 10 in $C$ ).

- The effect of the energy resolution was negligible for the size of the photon energy bins used. The absolute energy scale for the electromagnetic calorimeters is very well calibrated using Bhabha scattering events and cross-checked with, for example, the $\pi^{0}$ mass peak position.

- The background of calorimeter clusters from $\tau^{+} \tau^{-}$events in the selected sample (estimated with KORALZ 4.0 [28]) was less than $0.5 \%$ of the observed photon signal.

- The contamination from clusters produced by the LEP accelerator background or cosmic rays, estimated with the events collected using a random beam-crossing trigger, was below the level of 2 clusters per million events and no cluster passed the selection criteria.

To summarise, the principal systematic error comes from the uncertainty on the fitted fraction of photons in the data due to the uncertainty on the quality of the $\mathrm{MC}$ reproduction of the $C$ variable (point 1). The uncertainties due to the possible dependence of our result on the modelling of the prompt photon radiation, parton shower and hadronisation in the MC generators were estimated in points 2 and 3 . The total systematic uncertainty was calculated as the sum in quadrature of the propagated errors listed in points 1,2 and 3, as shown in Tab. 3. The systematic errors were partly correlated between the different photon energy ranges.

\section{Summary and discussion}

The energy spectrum of prompt photons in hadronic $\mathrm{Z}^{0}$ decays, corrected for the geometrical acceptance as discussed in section 5, is shown in Tab. 2 and in Fig. 6. The inner error bars are statistical, and the outer combined statistical and systematic (added in quadrature). The breakdown of the systematic uncertainties is given in Tab. 3 .

To compare our result with leading-order theoretical predictions for the quark-to-photon fragmentation function we use the leading-order cross-section for prompt photon production in $\mathrm{e}^{+} \mathrm{e}^{-}$annihilation given by formula (12) in [19]:

$$
\frac{1}{\sigma_{\text {had }}} \frac{d \sigma\left(E_{\gamma}\right)}{d E_{\gamma}}=\frac{4}{\sqrt{s}} \sum_{\mathrm{q}} w_{\mathrm{q}} D_{\gamma / \mathrm{q}}
$$

where $E_{\gamma}$ is the photon energy; $\sqrt{s}$ is the $\mathrm{e}^{+} \mathrm{e}^{-}$centre of mass energy; $w_{\mathrm{q}}$ is the relative contribution from quark flavour $\mathrm{q}\left(w_{\mathrm{q}}=\Gamma_{\mathrm{Z} \rightarrow \mathrm{q}} / \Gamma_{\mathrm{Z} \rightarrow \text { hadrons }}\right) ; \sigma_{\text {had }}$ is the production cross-section for $\mathrm{e}^{+} \mathrm{e}^{-}$hadronic events, and $D_{\gamma / \mathrm{q}}$ is the quark q to photon fragmentation function. In Fig. 6 we plot the QCD prediction using an asymptotic leading-order $D_{\gamma / \mathrm{q}}$ [6, 7] as parametrised by Duke and Owens [8] with $Q^{2}=M_{Z}^{2}$ and $\Lambda=0.2 \mathrm{GeV}$. In Fig. [6 we also plot the QCD predictions using $D_{\gamma / \mathrm{q}}$ from leading-order (LO) calculations by Glück, Reya and Vogt [9]. We also show the higher-order $(\mathrm{HO})$ prediction for the prompt photon production cross-section 
by Glück, Reya and Vogt [9] and the beyond leading logarithm (BLL) prediction by Bourhis, Fontannaz and Guillet [10]. The factorisation schemes used were DIS $_{\gamma}$ and $\overline{\mathrm{MS}}$ respectively. The authors of [9] and [10] included in their calculations non-perturbative effects through the vector-meson dominance ansatz, although using different experimental inputs.

Our data are in agreement with these theoretical predictions. The experimental precision is not sufficient to discriminate between them. The ALEPH data on the production of jets containing a photon carrying a substantial fraction (above 70\%) of the jet energy (Fig. 4 in [18]) show clear disagreement with the Duke-Owens parametrisation [8]. It was noted in [10] that a possible reason is that the ALEPH measurement is restricted to only part of the phasespace for photon production in hadronic $\mathrm{Z}^{0}$ decays.

To summarise, we have measured the inclusive production of prompt photons with energy above $10 \mathrm{GeV}$ in hadronic $\mathrm{Z}^{0}$ decays. Good agreement is found with current QCD predictions for the quark-to-photon fragmentation function.

\section{Acknowledgements}

We are grateful to Zoltan Kunszt, Werner Vogelsang, Richard Roberts and Nigel Glover for stimulating discussions on the theoretical aspects of our measurement. We also thank M. Glück, E. Reya, and A. Vogt, as well as L. Bourhis, M. Fontannaz, and J.Ph. Guillet for providing the numerical results of their calculations.

We particularly wish to thank the SL Division for the efficient operation of the LEP accelerator at all energies and for their continuing close cooperation with our experimental group. We thank our colleagues from CEA, DAPNIA/SPP, CE-Saclay for their efforts over the years on the time-of-flight and trigger systems which we continue to use. In addition to the support staff at our own institutions we are pleased to acknowledge the

Department of Energy, USA,

National Science Foundation, USA,

Particle Physics and Astronomy Research Council, UK,

Natural Sciences and Engineering Research Council, Canada,

Israel Science Foundation, administered by the Israel Academy of Science and Humanities, Minerva Gesellschaft, Benoziyo Center for High Energy Physics,

Japanese Ministry of Education, Science and Culture (the Monbusho) and a grant under the Monbusho International Science Research Program, German Israeli Bi-national Science Foundation (GIF), Bundesministerium für Bildung, Wissenschaft, Forschung und Technologie, Germany, National Research Council of Canada, Hungarian Foundation for Scientific Research, OTKA T-016660, T023793 and OTKA F023259. 


\section{References}

[1] CELLO Collab., H.J. Behrend et al., Z.Phys.C14 (1982) 189, CELLO Collab., H.J. Behrend et al., Z.Phys.C20 (1983) 207.

[2] JADE Collab., D.D. Pitzl et al., Z.Phys.C46 (1990) 1, (err.ibid. 47 (1990) 676),

JADE Collab., W. Bartel et al., Z.Phys.C28 (1985) 343.

[3] MAC Collab., E. Fernandez et al., Phys. Rev. Lett. 54 (1985) 95.

[4] TASSO Collab., W. Braunschweig et al., Z.Phys.C41 (1988) 385.

[5] VENUS Collab., K. Abe et al., Phys. Rev. Lett. 63 (1989) 1776.

[6] E. Witten, Nucl. Phys. B120 (1977) 189.

[7] C.H. Llewellyn Smith, Phys. Lett. B79 (1978) 83.

[8] J.F. Owens, Reviews of Modern Physics 59 (1987) 465,

D.W. Duke, J.F. Owens, Phys. Rev. D26 (1982) 1600.

[9] M. Glück, E. Reya, A. Vogt, Phys. Rev. D48 (1993) 116.

[10] L. Bourhis, M. Fontannaz, J.Ph. Guillet, preprint hep-ph 9704447.

[11] OPAL Collab., M.Z. Akrawy et al., Phys. Lett. B246 (1990) 285.

[12] E. Laermann, T.F. Walsh, I. Schmitt, P.M. Zerwas, Nucl. Phys. B207 (1982) 205 P. Mättig, W. Zeuner, Z.Phys.C52 (1991) 37.

[13] ALEPH Collab., D. Decamp et al., Phys. Lett. B264 (1991) 476, ALEPH Collab., D. Buskulic et al., Zeit. Phys.C57 (1993) 17.

[14] DELPHI Collab., P. Abreu et al., Zeit. Phys.C53 (1992) 555, DELPHI Collab., P. Abreu et al., Zeit. Phys.C69 (1995) 1.

[15] L3 Collab., O. Adriani et al., Phys. Lett. B292 (1992) 472, L3 Collab., O. Adriani et al., Phys. Lett. B297 (1993) 469, L3 Collab., O. Adriani et al., Phys. Lett. B301 (1993) 136, L3 Collab., M. Acciarri et al., Phys. Lett. B388 (1996) 409.

[16] OPAL Collab., G. Alexander et al., Phys. Lett. B264 (1991) 219, OPAL Collab., P.D. Acton et al., Zeit. Phys.C54 (1992) 193, OPAL Collab., P.D. Acton et al., Zeit. Phys.C58 (1993) 405, OPAL Collab., R. Akers et al., Zeit. Phys.C67 (1995) 15, OPAL Collab., R. Akers et al., Zeit. Phys.C68 (1995) 531, OPAL Collab., G. Alexander et al., Zeit. Phys.C71 (1996) 1.

[17] E.W.N. Glover, A.G. Morgan, Z.Phys.C62 (1994) 311. 
[18] ALEPH Collab.,D. Buskulic et al., Z.Phys.C69 (1996) 365.

[19] Z. Kunszt, Z. Trócsányi, Nucl. Phys. B394 (1993) 139.

[20] OPAL Collab., K. Ahmet et al., Nucl. Instrum. Methods A 305 (1991) 275.

[21] OPAL Collab., G. Alexander et al., Z.Phys.C52 (1991) 175.

[22] T. Sjöstrand, Comput. Phys. Commun. 39 (1986) 347, T. Sjöstrand, M.Bengtsson, Comput. Phys. Commun. 43 (1987) 43.

[23] G. Marchesini et al., Comput. Phys. Commun. 67 (1992) 465.

[24] OPAL Collab., G. Alexander et al., Z.Phys.C69 (1996) 543.

[25] J. Allison et al., Nucl. Instrum. Methods A 317 (1992) 47.

[26] OPAL Collab., R. Akers et al., Phys. Lett. B328 (1994) 207.

[27] R. Barlow, J. Comp. Phys. 72 (1987) 202, R. Barlow, C .Beeston, Comput. Phys. Commun. 77 (1993) 219.

[28] Z. Jadach, B. Ward, Z. Was, Comput. Phys. Commun. 79 (1994) 503.

[29] ALEPH Collab., D. Buskulic et al., Phys. Lett. B292 (1992) 210. 
Table 1: Energy dependent cuts and corrections to the photon energy spectrum. Corrections for photon efficiency and environment are multiplicative, ISR is subtracted.

\begin{tabular}{|c|c|c||c|l||l|}
\hline$E, \mathrm{GeV}$ & $\begin{array}{l}\Delta_{\text {cut }} \\
\mathrm{mrad}\end{array}$ & $S_{\text {cut }}$ & $\begin{array}{l}\text { isolated photon } \\
\text { efficiency }\end{array}$ & $\begin{array}{l}\text { photon } \\
\text { environment }\end{array}$ & $\begin{array}{l}N_{\gamma}^{I S R} / \mathrm{GeV} / 10^{6} \\
\text { events }\end{array}$ \\
\hline $10-15$ & 3. & 1.5 & $2.51 \pm 0.13$ & $1.34 \pm 0.10$ & $6.71 \pm 0.13$ \\
\hline $15-20$ & 4. & 2.5 & $2.06 \pm 0.12$ & $1.36 \pm 0.11$ & $2.62 \pm 0.08$ \\
\hline $20-25$ & 5. & 4. & $1.51 \pm 0.07$ & $1.37 \pm 0.10$ & $1.52 \pm 0.06$ \\
\hline $25-30$ & 5. & 5. & $1.47 \pm 0.08$ & $1.25 \pm 0.09$ & $1.27 \pm 0.06$ \\
\hline $30-35$ & 5. & 5. & $1.42 \pm 0.08$ & $1.20 \pm 0.09$ & $1.28 \pm 0.06$ \\
\hline $35-40$ & 5. & 5. & $1.60 \pm 0.09$ & $1.14 \pm 0.10$ & $1.20 \pm 0.06$ \\
\hline $40-45.6$ & 5. & 5. & $1.57 \pm 0.05$ & $1.03 \pm 0.13$ & $2.87 \pm 0.09$ \\
\hline
\end{tabular}

Table 2: The fraction $f$ of prompt photons in the selected sample and the corrected energy spectrum of prompt photons in hadronic $\mathrm{Z}^{0}$ events (the first error is statistical, the second systematic).

\begin{tabular}{|c|c|c|ccc|}
\hline$E, \mathrm{GeV}$ & Mean $E, \mathrm{GeV}$ & $f, \%$ & \multicolumn{3}{|c|}{$N_{\gamma} / \mathrm{GeV} / 10^{6}$ events } \\
\hline $10-15$ & 12.0 & $32.7 \pm 2.1$ & 625 & \pm 70 & \pm 99 \\
\hline $15-20$ & 17.3 & $18.2 \pm 1.7$ & 266 & \pm 36 & \pm 63 \\
\hline $20-25$ & 22.3 & $12.9 \pm 1.4$ & 142 & \pm 20 & \pm 37 \\
\hline $25-30$ & 27.3 & $27.9 \pm 1.9$ & 212 & \pm 24 & \pm 39 \\
\hline $30-35$ & 32.3 & $25.7 \pm 2.8$ & 118 & \pm 17 & \pm 28 \\
\hline $35-40$ & 37.1 & $29.9 \pm 5.0$ & 75 & \pm 15 & \pm 20 \\
\hline $40-45.6$ & 42.1 & $80.4 \pm 10.2$ & 48 & \pm 9 & \pm 8 \\
\hline
\end{tabular}


Table 3: Contributions to the systematic error on the photon energy spectrum shown in Tab. 2.

\begin{tabular}{|c|c||c|c|c|}
\hline \multirow{2}{*}{ Mean $E, \mathrm{GeV}$} & \multirow{2}{*}{$N_{\gamma} / \mathrm{GeV} / 10^{6}$ events } & \multicolumn{3}{|c|}{ Systematic errors on $N_{\gamma} / \mathrm{GeV} / 10^{6}$ events } \\
\cline { 3 - 5 } & $625 \pm 70 \pm 99$ & $\begin{array}{l}\text { description of } \\
C \text { variable }\end{array}$ & $\begin{array}{l}\text { model dependence } \\
\text { of background } \\
\text { distribution }\end{array}$ & photon acceptance \\
\hline 12.0 & $266 \pm 36 \pm 63$ & \pm 60 & \pm 73 & \pm 29 \\
\hline 17.3 & $142 \pm 20 \pm 37$ & \pm 32 & \pm 17 & \pm 12 \\
\hline 22.3 & $212 \pm 24 \pm 39$ & \pm 28 & \pm 25 & \pm 6 \\
\hline 27.3 & $118 \pm 17 \pm 28$ & \pm 23 & \pm 14 & \pm 6 \\
\hline 32.3 & $75 \pm 15 \pm 20$ & \pm 17 & \pm 9 & \pm 4 \\
\hline 37.1 & $48 \pm 9 \pm 8$ & \pm 5 & \pm 6 & \pm 3 \\
\hline 42.1 & & & & \pm 10 \\
\hline
\end{tabular}



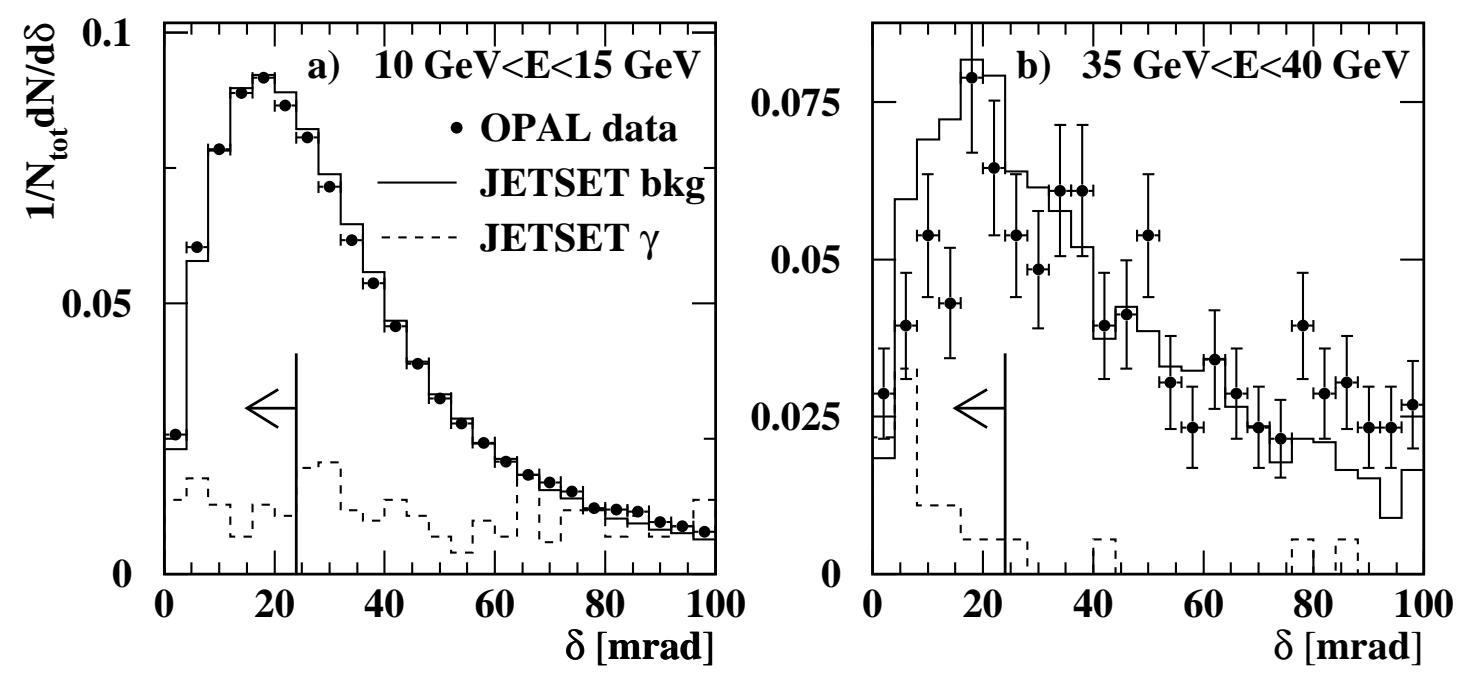

Figure 1: The angle, $\delta$, between the calorimeter cluster and the nearest track for hadronic events: for the data, background clusters in the JETSET simulated event sample and prompt photons in the JETSET sample, for different cluster energies: $10<E<15 \mathrm{GeV}$ a), and $35<E<40 \mathrm{GeV}$ b). Distributions are normalised such that the total yield for each curve is unity. Most of the photon signal is off scale, with $\delta>100 \mathrm{mrad}$. The regions removed by the cut are shown by arrows. 
Monte Carlo simulation
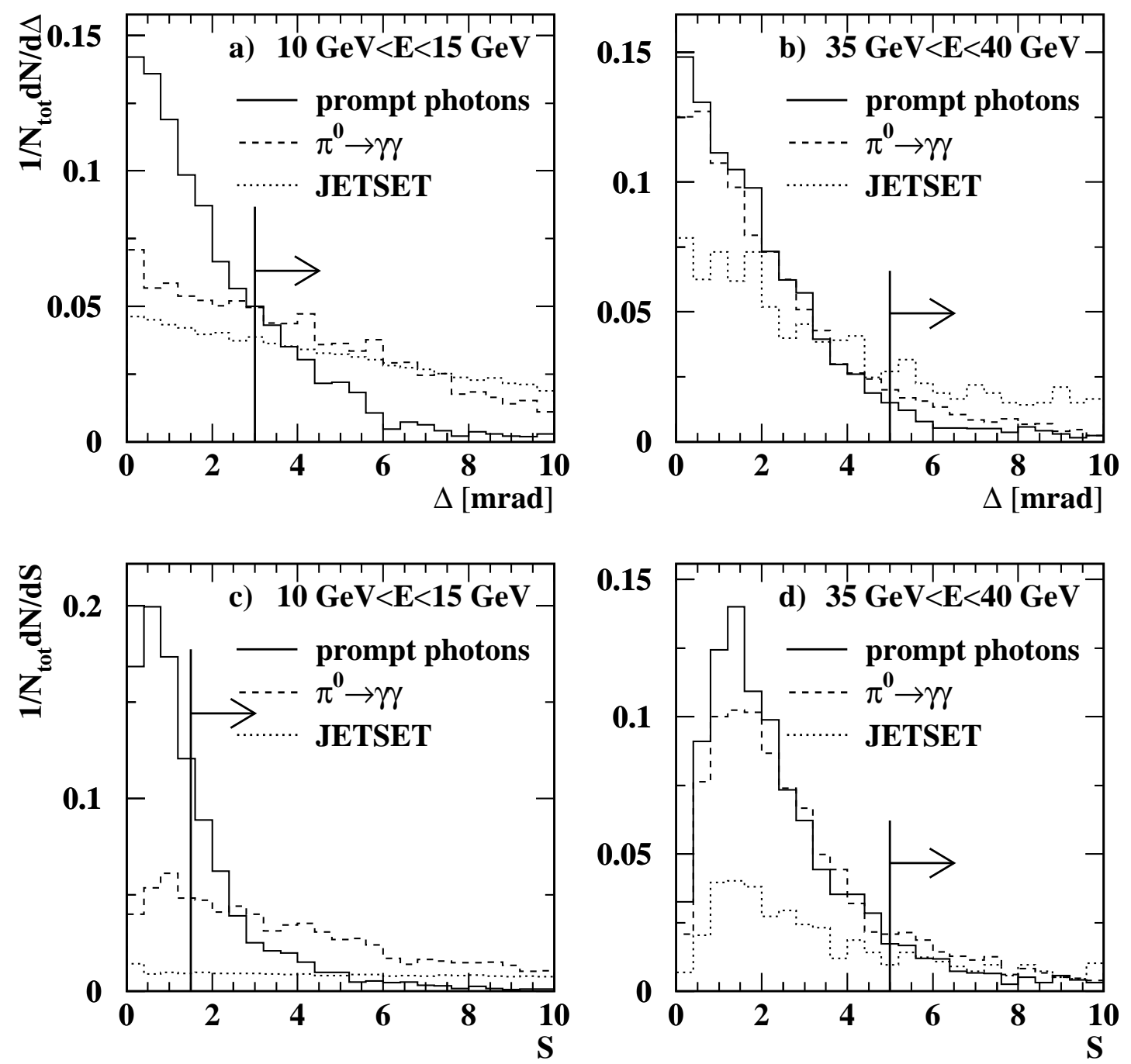

Figure 2: The distributions of the variables $\Delta$ (a and b), and $\mathrm{S}$ (c and d) for clusters from simulation of single isolated photons and $\pi^{0}$ 's and for all background clusters in JETSET events (after cut 1), for different cluster energies: $10<E<15 \mathrm{GeV}$ a) and c), and $35<E<40 \mathrm{GeV} \mathrm{b)} \mathrm{and} \mathrm{d).} \mathrm{Distributions} \mathrm{are} \mathrm{normalised} \mathrm{such} \mathrm{that} \mathrm{the} \mathrm{total} \mathrm{yield}$ for each curve is unity. The regions removed by the cuts are shown by arrows. 

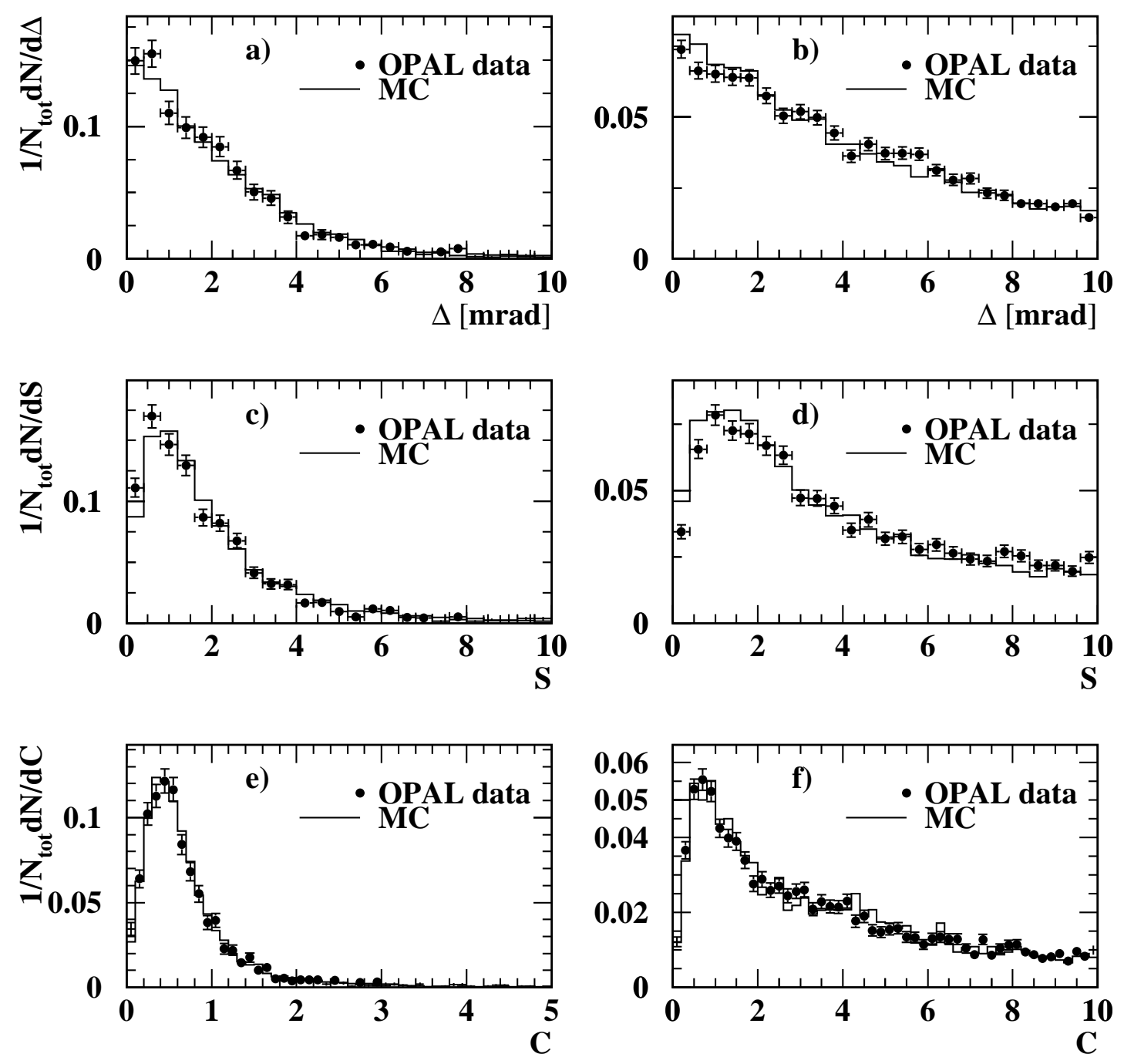

Figure 3: The distributions of the variables $\Delta$ (a and b), $S$ (c and d) and $C$ (e and $\mathrm{f}$ ) for photons in radiative lepton pair data events and simulated single, isolated photons (a, c and e); and $\pi^{0}$ 's in $\tau^{ \pm} \rightarrow \rho^{ \pm} \nu_{\tau}, \rho^{ \pm} \rightarrow \pi^{ \pm} \pi^{0}$ decays (b, d and f). Distributions are normalised such that the total yield for each curve is unity 

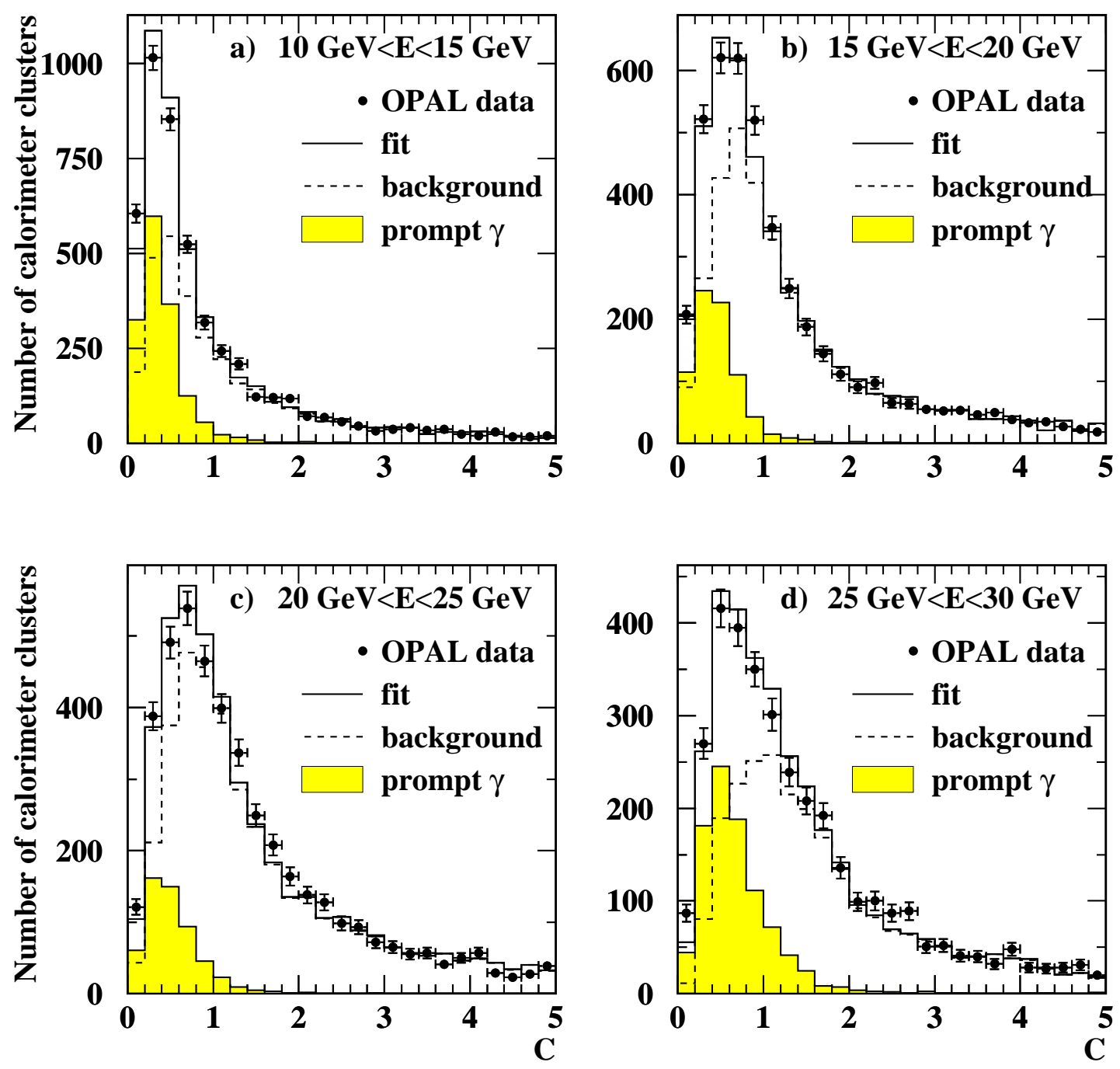

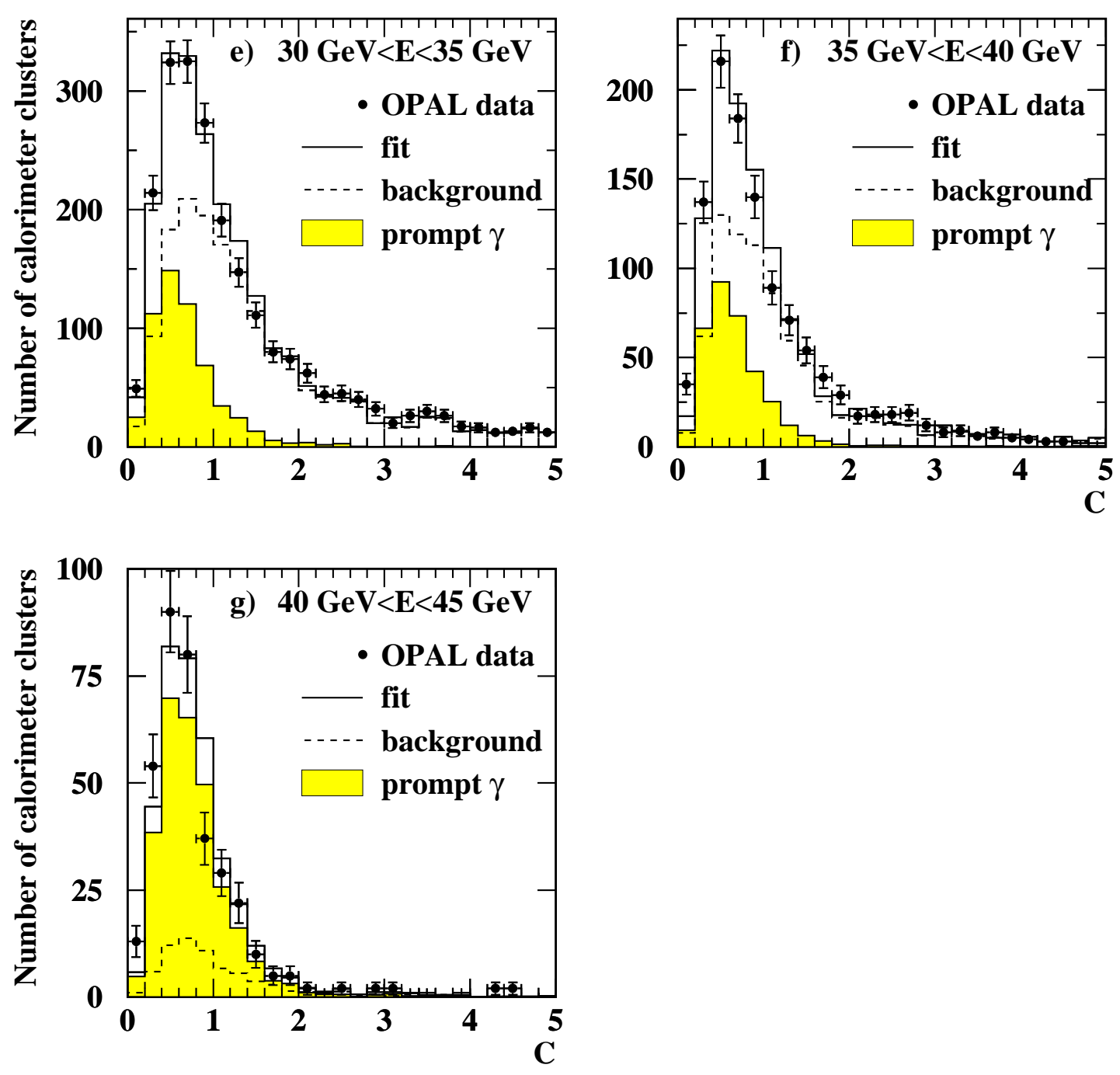

Figure 4: Comparison of the data and fit for different cluster energy ranges. Contributions from prompt photons and background in the fit are shown. 


\section{Monte Carlo simulation}
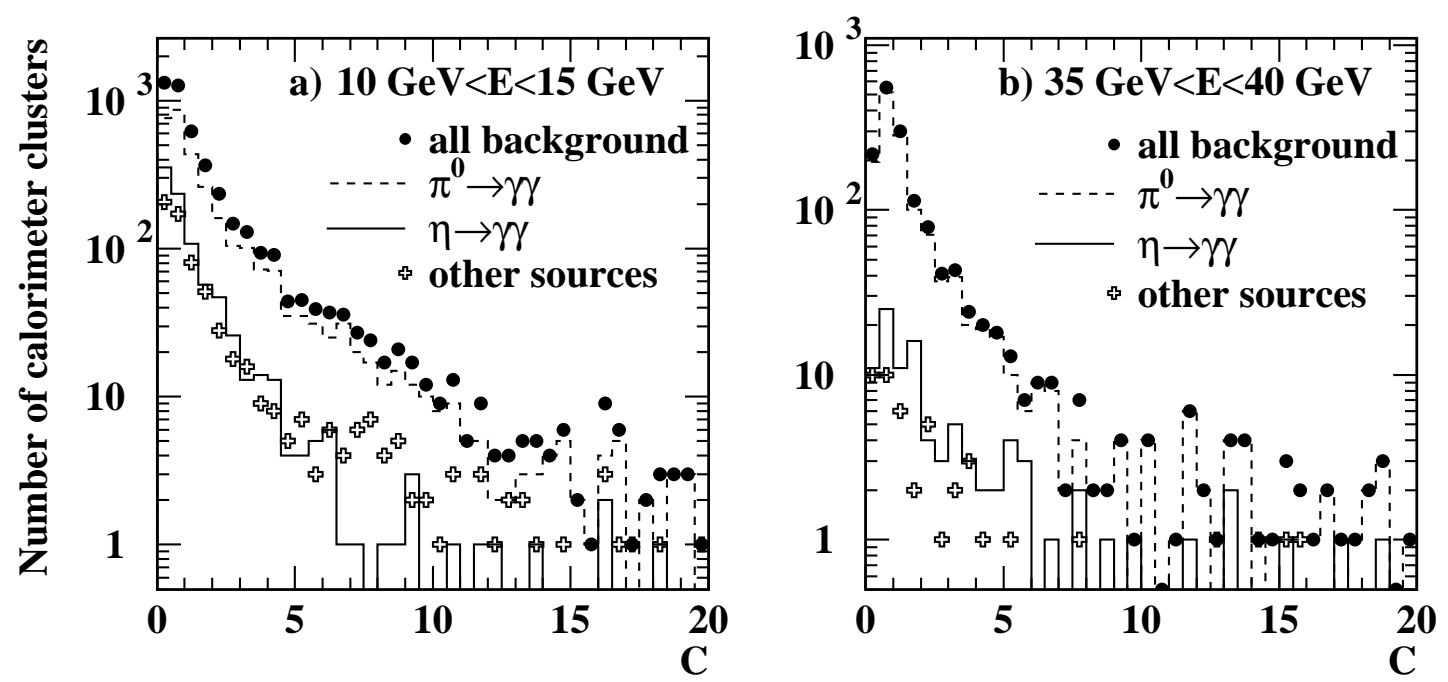

Figure 5: $C$ variable distributions for background clusters from different sources in the JETSET simulation for different cluster energy ranges. 


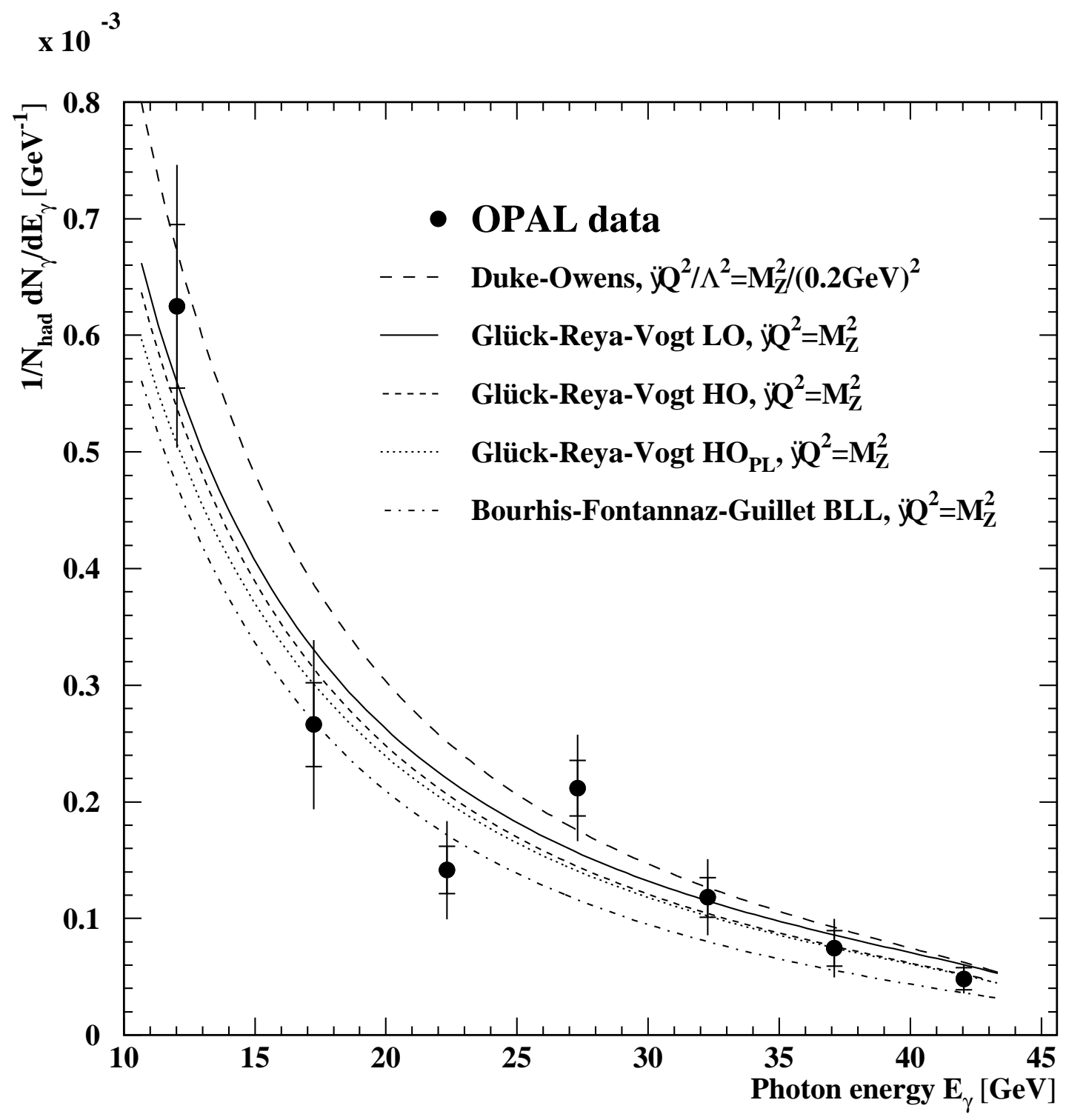

Figure 6: The photon energy spectrum in hadronic $\mathrm{Z}^{0}$ decays compared to various theoretical predictions: the Duke-Owens parametrisation [8], the Glück, Reya and Vogt predictions including leading-order (LO), higher-order (HO) and higher-order without the non-perturbative corrections $\left(\mathrm{HO}_{\mathrm{PL}}\right)$ [9]. The Bourhis, Fontannaz and Guillet prediction shown include effects beyond leading logarithms (BLL) [10]. 


\title{
AXODRAW
}

\author{
J.A.M.Vermaseren \\ NIKHEF-H \\ P.O. Box 41882 \\ 1009 DB Amsterdam
}

\begin{abstract}
Axodraw is a set of drawing primitives for use in $\mathrm{I}_{\mathrm{E}} \mathrm{TX}$. These can be used for the drawing of Feynman diagrams, flow charts and simple graphics. Because it uses postscript for its drawing commands it works only in combination with the dvips of Radical Eye Software which is presently the most popular dvips program. More will be added in the future. It allows whole articles including their pictures to be contained in a single file, thereby making it easier to exchange the article file by e-mail.
\end{abstract}




\section{Using Axodraw}

The file axodraw.sty is a style file for $\mathrm{LAT}_{\mathrm{E}} \mathrm{X}$. It should be included in the documentstyle statement at the beginning of the document. An example would be:

\documentstyle [a4,11pt, axodraw $\{$ article\}

Because axodraw.sty reads also the epsf.sty file that comes with many implementations of $\mathrm{T}_{\mathrm{E} X}$ and in particular those that rely on the dvips program by Radical Eye Software for the printing, this file should be present in the system. If this file is not available one should obtain it from another system. The author feels in no way responsible for the problems that may occur when a different dvi-to-postscript program is used.

The drawing is actually done in postscript. Because the above mentioned dvi-to-postscript converter allows the inclusion of postscript code the graphics primitives have been included in the file axodraw.sty in terms of postscript. If another postscript converter is used, one may have to adapt the syntax of the inclusion of this code to the local system.

The commands of Axodraw should be executed inside either the picture or the figure environment. Inside this environment it is possible to place objects at arbitrary positions and put text between them. In principle one could try to draw objects with the facilities of $\mathrm{LAT}_{\mathrm{E}} \mathrm{X}$ itself, but it turns out that the commands in the picture environment are not very powerful. Axodraw gives good extensions of them. An example would be

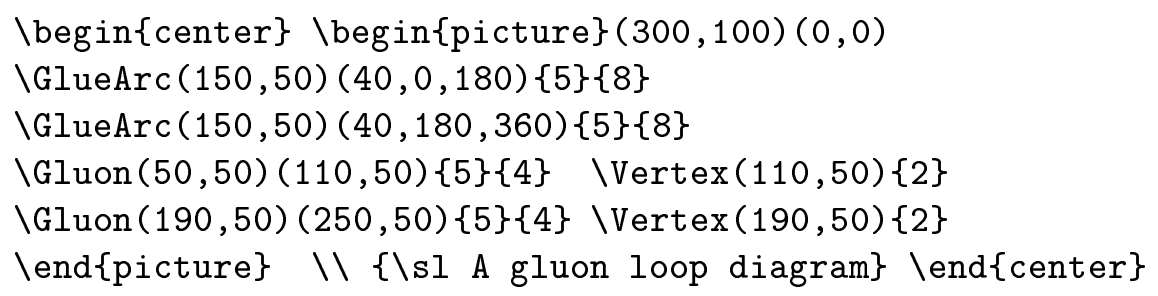

This code would result in:

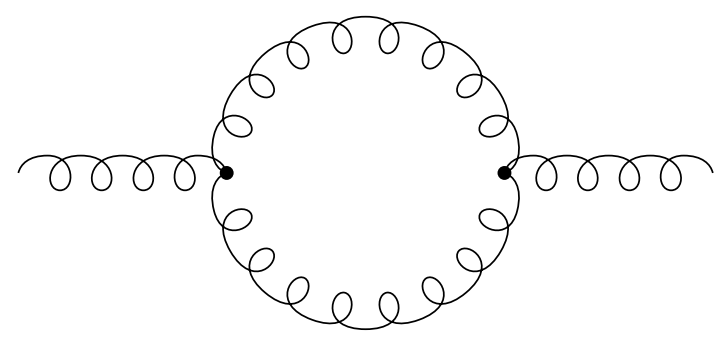

A gluon loop diagram

The syntax and the meaning of these command are explained in the next section. One should note that all coordinates are presented in units of 1 point. There are 72 points in an inch. It is possible to use scale transformations if these units are not convenient. 
Currently the primitives are mainly useful for the drawing of Feynman diagrams and the drawing of flowcharts. This means that the commands were designed to draw a number of these graphs. Of course many more things can be drawn with them, like scatter plots, histograms etc. With such use it may be though that the user will miss some primitives that would come in handy. Of course in future versions there will be more primitives, especially when users suggest some really good ones.

\section{The commands}

The commands that are currently available in Axodraw are (in alphabetic order):

- $\backslash \operatorname{Arrow} \operatorname{Arc}(\mathrm{x}, \mathrm{y})\left(\mathrm{r}, \phi_{1}, \phi_{2}\right)$

Draws an arc segment centered around $(\mathrm{x}, \mathrm{y})$. The radius is $\mathrm{r}$. The arcsegment runs counterclockwise from $\phi_{1}$ to $\phi_{2}$. All angles are given in degrees. In the middle of the segment there will be an arrow.

- $\backslash \operatorname{Arrow} \operatorname{Arcn}(\mathrm{x}, \mathrm{y})\left(\operatorname{radius}, \phi_{1}, \phi_{2}\right)$

Draws an arc segment centered around $(\mathrm{x}, \mathrm{y})$. The radius is $\mathrm{r}$. The arcsegment runs clockwise from $\phi_{1}$ to $\phi_{2}$. All angles are given in degrees. In the middle of the segment there will be an arrow.

- $\backslash$ ArrowLine $\left(x_{1}, y_{1}\right)\left(x_{2}, y_{2}\right)$

Draws a line from $\left(x_{1}, y_{1}\right)$ to $\left(x_{2}, y_{2}\right)$. There will be an arrow in the middle of the line.

- $\backslash \operatorname{BBox}\left(x_{1}, y_{1}\right)\left(x_{2}, y_{2}\right)$

Draws a box of which the contents are blanked out. This means that anything that was present at the position of the box will be overwritten. The lower left corner of the box is at $\left(x_{1}, y_{1}\right)$ and $\left(x_{2}, y_{2}\right)$ is the upper right corner of the box.

- $\backslash \operatorname{BBoxc}(\mathrm{x}, \mathrm{y})$ (width, height)

Draws a box of which the contents are blanked out. This means that anything that was present at the position of the box will be overwritten. The center of the box is at $(\mathrm{x}, \mathrm{y})$. Width and height refer to the full width and the full height of the box.

- $\backslash \mathrm{BCirc}(\mathrm{x}, \mathrm{y})\{\mathrm{r}\}$

Draws a circle of which the contents are blanked out. This means that anything that was present at the position of the circle will be overwritten. The center of the circle is at $(\mathrm{x}, \mathrm{y}), \mathrm{r}$ is its radius.

- $\backslash \operatorname{Boxc}(\mathrm{x}, \mathrm{y})($ width,height)

Draws a box. The center of the box is at $(x, y)$. Width and height refer to the full width and the full height of the box. 
- $\backslash \mathrm{BText}(\mathrm{x}, \mathrm{y})\{$ text

Draws a box with one line of centered postscript text in it. The box is just big enough to fit around the text. The coordinates refer to the center of the box. The box is like a BBox in that it blanks out whatever was at the position of the box.

- $\backslash \mathrm{B} 2 \operatorname{Text}(\mathrm{x}, \mathrm{y})\{\operatorname{text} 1\}\{\operatorname{text} 2\}$

Draws a box with two lines of centered postscript text in it. The box is just big enough to fit around the text. The coordinates refer to the center of the box. The box is like a BBox in that it blanks out whatever was at the position of the box.

- $\backslash \operatorname{CArc}(\mathrm{x}, \mathrm{y})\left(\right.$ radius $\left., \phi_{1}, \phi_{2}\right)$

Draws an arc segment centered around $(\mathrm{x}, \mathrm{y})$. The radius is $\mathrm{r}$. The arcsegment runs counterclockwise from $\phi_{1}$ to $\phi_{2}$. All angles are given in degrees.

- \Curve $\left\{\left(x_{1}, y_{1}\right)\left(x_{2}, y_{2}\right) \cdots\left(x_{n}, y_{n}\right)\right\}$

Draws a curve through the given points. The $\mathrm{x}$-values are supposed to be in ascending order. The curve is a combination of quadratic and third order segments and is continuous in its first and second derivatives.

- $\backslash$ DashArrowArc $(\mathrm{x}, \mathrm{y})\left(\mathrm{r}, \phi_{1}, \phi_{2}\right)\{$ dashsize $\}$

Draws a dashed arc segment centered around $(\mathrm{x}, \mathrm{y})$. The radius is $\mathrm{r}$. The arc-segment runs counterclockwise from $\phi_{1}$ to $\phi_{2}$. All angles are given in degrees. In the middle of the segment there will be an arrow. The size of the dashes is approximately equal to 'dashsize'.

- \DashArrowArcn $(\mathrm{x}, \mathrm{y})\left(\right.$ radius $\left., \phi_{1}, \phi_{2}\right)\{$ dashsize $\}$

Draws a dashed arc segment centered around $(\mathrm{x}, \mathrm{y})$. The radius is $\mathrm{r}$. The arc-segment runs clockwise from $\phi_{1}$ to $\phi_{2}$. All angles are given in degrees. In the middle of the segment there will be an arrow. The size of the dashes is approximately equal to 'dashsize'.

- $\backslash$ DashArrowLine $\left(x_{1}, y_{1}\right)\left(x_{2}, y_{2}\right)\{$ dashsize $\}$

Draws a line from $\left(x_{1}, y_{1}\right)$ to $\left(x_{2}, y_{2}\right)$ with a dashed pattern. The size of the black parts of the pattern is given by 'dashsize'. The alternating pieces have equal length. The size of the pattern is adjusted so that both the begin and the end are black. Halfway the line there is an arrow.

- $\backslash \operatorname{DashCArc}(\mathrm{x}, \mathrm{y})\left(\operatorname{radius}, \phi_{1}, \phi_{2}\right)\{$ dashsize $\}$

Draws a dashed arc segment centered around $(\mathrm{x}, \mathrm{y})$. The radius is $\mathrm{r}$. The arc-segment runs counterclockwise from $\phi_{1}$ to $\phi_{2}$. All angles are given in degrees. The size of the dashes is determined by 'dashsize'. This size is adjusted somewhat to make the result look nice.

- $\backslash$ DashCurve $\left\{\left(x_{1}, y_{1}\right)\left(x_{2}, y_{2}\right) \cdots\left(x_{n}, y_{n}\right)\right\}\{$ dashsize $\}$

Draws a dashed curve through the given points. The $\mathrm{x}$-values are supposed to be in ascending order. The curve is a combination of quadratic and third order segments. The size of the black parts and the white parts 
will be approximately 'dashsize' each. Some adjustment takes place to make the pattern come out right at the endpoints.

- $\backslash$ DashLine $\left(x_{1}, y_{1}\right)\left(x_{2}, y_{2}\right)\{$ dashsize $\}$

Draws a line from $\left(x_{1}, y_{1}\right)$ to $\left(x_{2}, y_{2}\right)$ with a dashed pattern. The size of the black parts of the pattern is given by 'dashsize'. The alternating pieces have equal length. The size of the pattern is adjusted so that both the begin and the end are black.

- $\backslash \operatorname{EBox}\left(x_{1}, y_{1}\right)\left(x_{2}, y_{2}\right)$

Draws a box. The lower left corner of the box is at $\left(x_{1}, y_{1}\right)$ and $\left(x_{2}, y_{2}\right)$ is the upper right corner of the box.

- $\backslash \operatorname{GBox}\left(x_{1}, y_{1}\right)\left(x_{2}, y_{2}\right)\{$ grayscale $\}$

Draws a box. The lower left corner of the box is at $\left(x_{1}, y_{1}\right)$ and $\left(x_{2}, y_{2}\right)$ is the upper right corner of the box. The contents of the box are lost. They are overwritten with a color gray that is indicated by the parameter 'grayscale'. This parameter can have values ranging from 0 (black) to 1 (white).

- $\backslash \operatorname{GBoxc}(\mathrm{x}, \mathrm{y})($ width,height) $\{$ grayscale\}

Draws a box. The center of the box is at $(\mathrm{x}, \mathrm{y})$. Width and height refer to the full width and the full height of the box. The contents of the box are lost. They are overwritten with a color gray that is indicated by the parameter 'grayscale'. This parameter can have values ranging from 0 (black) to 1 (white).

- $\backslash \operatorname{GCirc}(\mathrm{x}, \mathrm{y})\{$ radius $\}$ grayscale\}

Draws a circle around $(\mathrm{x}, \mathrm{y})$ with radius $\mathrm{r}$. The contents of the circle are lost. They are overwritten with a color gray that is indicated by the parameter 'grayscale'. This parameter can have values ranging from 0 (black) to 1 (white).

- $\backslash$ GlueArc $(\mathrm{x}, \mathrm{y})\left(\mathrm{r}, \phi_{1}, \phi_{2}\right)\{$ amplitude $\}$ windings $\}$

Draws a gluon on an arc-segment. The center of the arc is $(\mathrm{x}, \mathrm{y})$ and $\mathrm{r}$ is its radius. The arc segment runs counterclockwise from $\phi_{1}$ to $\phi_{2}$. The width of the gluon is twice 'amplitude', and the number of windings is given by the last parameter. Note that whether the curls are inside or outside can be influenced with the sign of the amplitude. When it is positive the curls are on the inside.

- $\backslash \operatorname{Gluon}\left(x_{1}, y_{1}\right)\left(x_{2}, y_{2}\right)\{$ amplitude $\}\{$ windings $\}$

Draws a gluon from $\left(x_{1}, y_{1}\right)$ to $\left(x_{2}, y_{2}\right)$. The width of the gluon will be twice the value of 'amplitude'. The number of windings is given by the last parameter. If this parameter is not an integer it will be rounded to an integer value. The side at which the windings lie is determined by the order of the two coordinates. Also a negative amplitude can change this side. 
- $\backslash \operatorname{GOval}(\mathrm{x}, \mathrm{y})(\mathrm{h}, \mathrm{w})(\phi)\{$ grayscale $\}$ Draws an oval with an internal color indicated by grayscale. This parameter can have values ranging from 0 (black) to 1 (white). The center of the oval is given by $(\mathrm{x}, \mathrm{y})$. Its height is $\mathrm{h}$, and the width is w. In addition the oval can be rotated counterclockwise over $\phi$ degrees. The oval overwrites anything that used to be in its position.

- $\backslash \mathrm{GText}(\mathrm{x}, \mathrm{y})\{$ grayscale $\}$ text $\}$

Draws a gray box with one line of centered postscript text in it. The box is just big enough to fit around the text. The coordinates refer to the center of the box. The box is like a BBox in that it blanks out whatever was at the position of the box.

- \G2Text(x,y)\{grayscale $\}\{$ text 1$\}\{$ text2\}

Draws a gray box with two lines of centered postscript text in it. The box is just big enough to fit around the text. The coordinates refer to the center of the box. The box is like a BBox in that it blanks out whatever was at the position of the box.

- $\backslash \operatorname{LinAxis}\left(x_{1}, y_{1}\right)\left(x_{2}, y_{2}\right)\left(N_{D}, d\right.$, hashsize ,offset,width $)$

This draws a line to be used as an axis in a graph. Along the axis are hash marks. Going from the first coordinate to the second, the hash marks are on the left side if 'hashsize', which is the size of the hash marks, is positive and on the right side if it is negative. $N_{D}$ is the number of 'decades', indicated by fat hash marks, and $d$ is the number of subdivisions inside each decade. The offset parameter tells to which subdivision the first coordinate corresponds. When it is zero, this coordinate corresponds to a fat mark of a decade. Because axes have their own width, this is indicated with the last parameter.

- $\backslash$ Line $\left(x_{1}, y_{1}\right)\left(x_{2}, y_{2}\right)$

Draws a line from $\left(x_{1}, y_{1}\right)$ to $\left(x_{2}, y_{2}\right)$.

- $\backslash \log \operatorname{Axis}\left(x_{1}, y_{1}\right)\left(x_{2}, y_{2}\right)\left(N_{L}\right.$, hashsize ,offset,width) This draws a line to be used as an axis in a graph. Along the axis are hash marks. Going from the first coordinate to the second, the hash marks are on the left side if 'hashsize', which is the size of the hash marks, is positive and on the right side if it is negative. $N_{L}$ is the number of orders of magnitude, indicated by fat hash marks. The offset parameter tells to which integer subdivision the first coordinate corresponds. When it is zero, this coordinate corresponds to a fat mark, which is identical to when the value would have been 1 . Because axes have their own width, this is indicated with the last parameter.

- \LongArrow $\left(x_{1}, y_{1}\right)\left(x_{2}, y_{2}\right)$

Draws a line from $\left(x_{1}, y_{1}\right)$ to $\left(x_{2}, y_{2}\right)$. There will be an arrow at the end of the line. 
- $\backslash$ LongArrowArc(x,y) $\left(\mathrm{r}, \phi_{1}, \phi_{2}\right)$

Draws an arc segment centered around $(\mathrm{x}, \mathrm{y})$. The radius is $\mathrm{r}$. The arcsegment runs counterclockwise from $\phi_{1}$ to $\phi_{2}$. All angles are given in degrees. At the end of the segment there will be an arrow.

- \LongArrow $\operatorname{Arcn}(\mathrm{x}, \mathrm{y})\left(\right.$ radius $\left., \phi_{1}, \phi_{2}\right)$

Draws an arc segment centered around $(\mathrm{x}, \mathrm{y})$. The radius is $\mathrm{r}$. The arcsegment runs clockwise from $\phi_{1}$ to $\phi_{2}$. All angles are given in degrees. At the end of the segment there will be an arrow.

- $\backslash \operatorname{Oval}(\mathrm{x}, \mathrm{y})(\mathrm{h}, \mathrm{w})(\phi)$ Draws an oval. The center of the oval is given by $(\mathrm{x}, \mathrm{y})$. Its height is $\mathrm{h}$, and the width is $\mathrm{w}$. In addition the oval can be rotated counterclockwise over $\phi$ degrees. The oval does not overwrite its contents.

- $\backslash \operatorname{Photon}\left(x_{1}, y_{1}\right)\left(x_{2}, y_{2}\right)\{$ amplitude $\}$ wiggles $\}$ Draws a photon from $\left(x_{1}, y_{1}\right)$ to $\left(x_{2}, y_{2}\right)$. The width of the photon will be twice the value of 'amplitude'. The number of wiggles is given by the last parameter. If twice this parameter is not an integer it will be rounded to an integer value. Whether the first wiggle starts up or down can be influenced with the sign of the amplitude.

- $\backslash$ Photon $\operatorname{Arc}(\mathrm{x}, \mathrm{y})\left(\mathrm{r}, \phi_{1}, \phi_{2}\right)\{$ amplitude $\}$ wiggles $\}$

Draws a photon on an arc-segment. The center of the arc is $(\mathrm{x}, \mathrm{y})$ and $\mathrm{r}$ is its radius. The arc segment runs counterclockwise from $\phi_{1}$ to $\phi_{2}$. The width of the photon is twice 'amplitude', and the number of wiggles is given by the last parameter. Note that the sign of the amplitude influences whether the photon starts going outside (positive) or starts going inside (negative). If one likes the photon to reach both endpoints from the outside the number of wiggles should be an integer plus 0.5.

- $\backslash \mathrm{PText}(\mathrm{x}, \mathrm{y})(\phi)[\operatorname{mode}]\{$ text $\}$

Places a postscript text. The focal point is $(\mathrm{x}, \mathrm{y})$. The text is the last parameter. The mode parameter tells how the text should be positioned with respect to the focal point. If this parameter is omitted the center of the text will correspond to the focal point. Other options are: 1 for having the left side correspond to the focal point, $r$ for having the right side correspond to it, $\mathrm{t}$ for having the top at the focal point and $\mathrm{b}$ for the bottom. One may combine two letters as in [bl], as long as it makes sense. The parameter $\phi$ is a rotation angle. The text is written in the current postscript font. This font can be set with the SetPFont command.

- $\backslash \mathrm{rText}(\mathrm{x}, \mathrm{y})[$ mode] [rotation] $\{$ text $\}$

Places a rotated text. The focal point is $(x, y)$. The text is the last parameter. If the rotation parameter is the character 1 the text will be rotated left by 90 degrees, if it is an $\mathrm{r}$ it will be rotated to the right by 90 degrees and when it is the character $u$ the text will be rotated by 180 degrees. When there is no character there is no rotation and the command is identical to the Text command. The mode parameter tells 
how the resulting box should be positioned with respect to the focal point. If this parameter is omitted the center of the box will correspond to the focal point. Other options are: 1 for having the left side correspond to the focal point, $\mathrm{r}$ for having the right side correspond to it, $\mathrm{t}$ for having the top at the focal point and $\mathrm{b}$ for the bottom. One may combine two letters as in [bl], as long as it makes sense.

- $\backslash$ SetPFont $\{$ fontname $\}$ fontsize $\}$

Sets the postscript font to a given type and scale.

- $\backslash$ SetScale\{scalevalue\}

Changes the scale of all graphics operations. Unfortunately it does not change the scale of the text operations (yet?). A 'scalevalue' of 1 is the default. It is allowed to use floating point values.

- \SetOffset(x_offset,y_offset)

Adds the offset values to all coordinates at the $\mathrm{T}_{\mathrm{EX}}$ level. This makes it easier to move figures around.

- \SetScaledOffset(x_offset,y_offset)

Adds the offset values to all coordinates at the postscript level. This is done after scaling has been applied. Hence one can work with the scaled coordinates. This can be very handy when drawing curves.

- \SetWidth\{widthvalue\}

Changes the linewidth in all graphics operations. It does not change the linewidth of the text operations. That is a matter of font selection. A 'widthvalue' of 0.5 is the default. It is allowed to use floating point values.

- $\backslash \operatorname{Text}(\mathrm{x}, \mathrm{y})[\operatorname{mode}]\{$ text $\}$

Places a text. The focal point is $(\mathrm{x}, \mathrm{y})$. The text is the last parameter. The mode parameter tells how the text should be positioned with respect to the focal point. If this parameter is omitted the center of the text will correspond to the focal point. Other options are: 1 for having the left side correspond to the focal point, $\mathrm{r}$ for having the right side correspond to it, $\mathrm{t}$ for having the top at the focal point and $\mathrm{b}$ for the bottom. One may combine two letters as in [bl], as long as it makes sense.

- $\backslash \operatorname{Vertex}(\mathrm{x}, \mathrm{y})\{\mathrm{r}\}$

Draws a fat dot at $(\mathrm{x}, \mathrm{y})$. The radius of the dot is given by $\mathrm{r}$.

- $\backslash \operatorname{ZigZag}\left(x_{1}, y_{1}\right)\left(x_{2}, y_{2}\right)\{$ amplitude $\}$ wiggles $\}$

Draws a zigzag line from $\left(x_{1}, y_{1}\right)$ to $\left(x_{2}, y_{2}\right)$. The width of the zigzagging will be twice the value of 'amplitude'. The number of zigzags is given by the last parameter. If twice this parameter is not an integer it will be rounded to an integer value. Whether the first zigzag starts up or down can be influenced with the sign of the amplitude. 


\section{Examples}

Although the previous section contains all the commands and their proper syntax a few examples may be helpful.

\subsection{Text modes}

The meaning of the mode characters in the text command can best be demonstrated. The statements

$$
\begin{aligned}
& \backslash \text { begin\{center\} \begin\{picture } ( 3 0 0 , 1 0 0 ) ( 0 , 0 ) }\{center\} \begin\{picture} ( 3 0 0 , 1 0 0 ) ( 0 , 0 ) } \\
{\backslash \operatorname{CArc}(50,75)(2,0,360) \quad \backslash \operatorname{Text}(50,75)[1 \mathrm{t}]\{\text { left-top\} }} \\
{\backslash \operatorname{CArc}(50,50)(2,0,360) \quad \backslash \operatorname{Text}(50,50)[1]\{\text { left-center\} }} \\
{\backslash \operatorname{CArc}(50,25)(2,0,360) \quad \backslash \operatorname{Text}(50,25)[1 \mathrm{~b}]\{\text { left-bottom\} }} \\
{\backslash \operatorname{CArc}(150,75)(2,0,360) \backslash \operatorname{Text}(150,75)[\mathrm{t}] \text { \{center-top\} }} \\
{\backslash \operatorname{CArc}(150,50)(2,0,360) \backslash \operatorname{Text}(150,50)[] \text { \{center-center\} }} \\
{\backslash \operatorname{CArc}(150,25)(2,0,360) \backslash \operatorname{Text}(150,25)[\mathrm{b}] \text { \{center-bottom\} }} \\
{\backslash \operatorname{CArc}(250,75)(2,0,360) \backslash \operatorname{Text}(250,75)[\mathrm{rt}] \text { \{right-top\} }} \\
{\backslash \operatorname{CArc}(250,50)(2,0,360) \backslash \operatorname{Text}(250,50)[\mathrm{r}] \text { \{right-center\} }} \\
{\backslash \operatorname{CArc}(250,25)(2,0,360) \backslash \operatorname{Text}(250,25)[\mathrm{rb}] \text { \{right-bottom\} }} \\
{\text { lend\{picture\} lend } \text { center\} }}
\end{aligned}
$$

produce 9 texts and for each the focal point is indicated by a little circle. It looks like

$$
\begin{array}{lcr}
\text { Qeft-top } & \text { center-top } & \text { right-top } \\
\text { qeft-center } & \text { centerøcenter } & \text { right-centero } \\
\text { deft-bottom } & \text { center-bottom right-bottomo }
\end{array}
$$

This illustrates exactly all the combinations of the mode characters and what their effects are. The text can of course contain different fonts, math mode and all those little things that are usually easier in $\mathrm{AT}_{\mathrm{E}} \mathrm{X}$ than in postscript.

\subsection{The windings of a gluon}

Gluons are traditionally represented by a two dimensional projection of a helix. Actually close inspection of some pretty gluons reveals that it is usually not quite a helix. Hence the gluons in Axodraw are also not quite helices. In addition one may notice that the begin and end points deviate slightly from the regular windings. This makes it more in agreement with hand drawn gluons. When a gluon is drawn, one needs not only its begin and end points but there is an amplitude connected to this almost helix, and in addition there are windings. The number of windings is the number of curls that the gluon will have. Different people may prefer different densities of curls. This can effect the appearance considerably: 


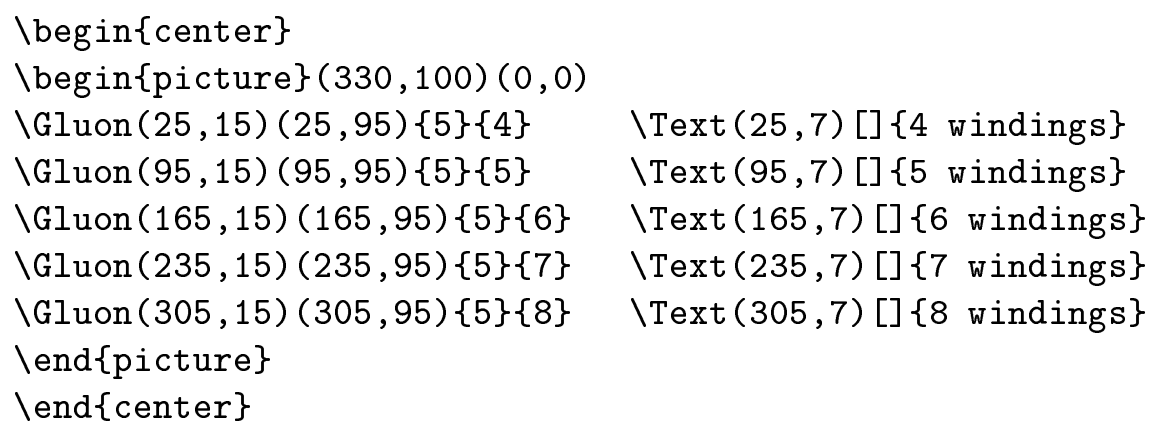

This code results in:

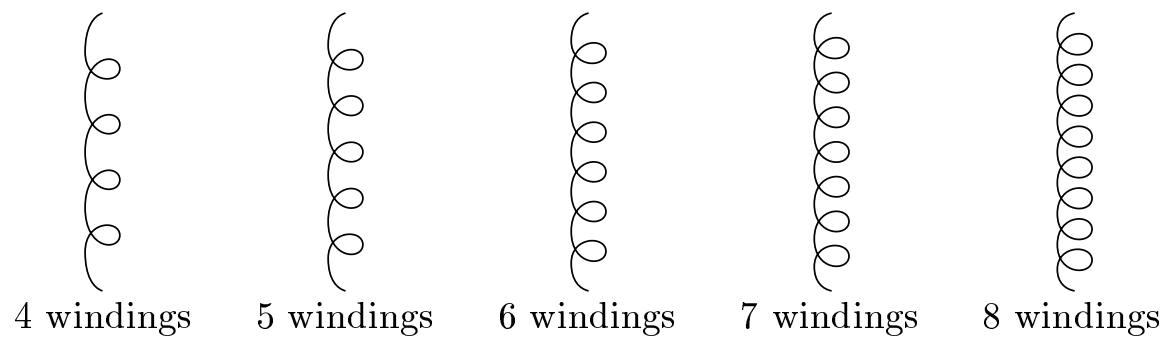

The influence of the amplitude is also rather great. The user should experiment with it. There is however an aspect to the amplitude that should be discussed. For a straight gluon the amplitude can determine on which side the curls are. So does the direction of the gluon:

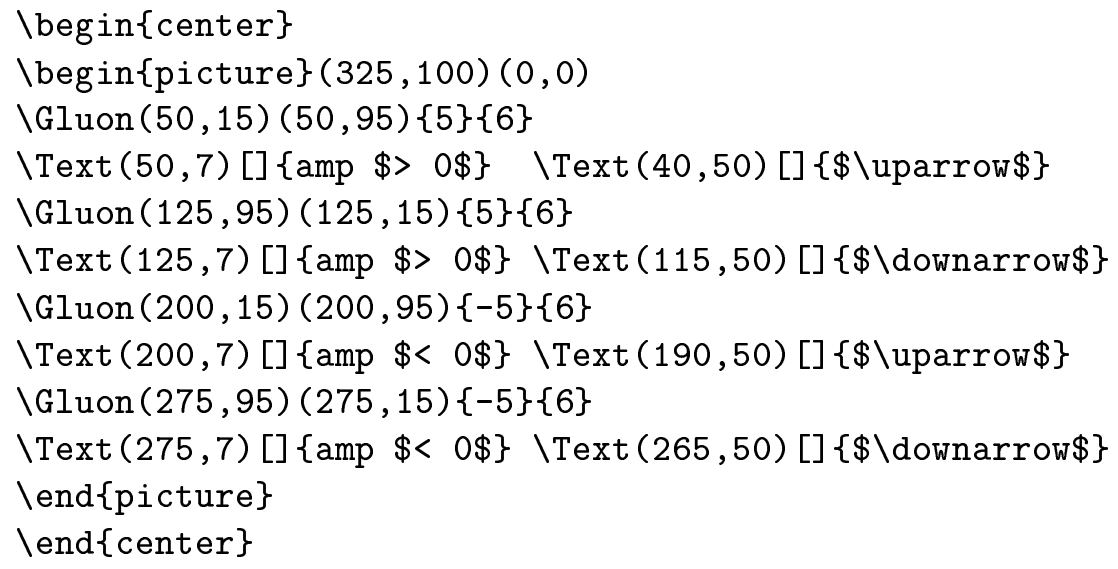

The picture gets the following appearance:
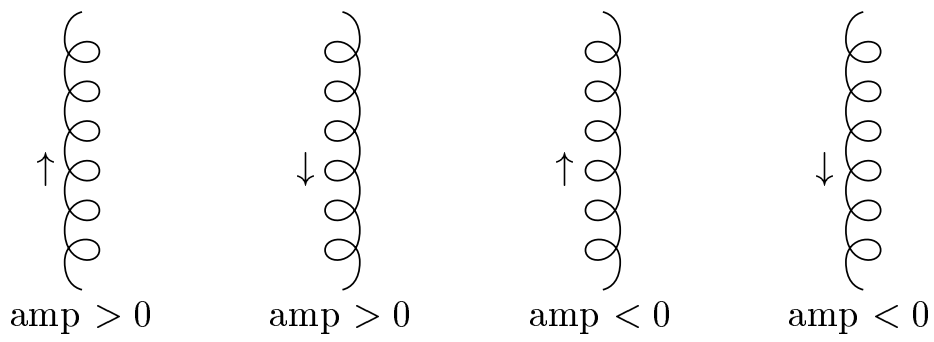
For straight gluons one does not need the option of the negative amplitude. It is however necessary for gluons on an arc segment. In that case the arc is always drawn in an anticlockwise direction. Hence the direction is fixed and only the amplitude is left as a tool for determining the side with the curls.

\subsection{Scaling}

Sometimes it is much easier to design a figure on a larger scale than it is needed in the eventual printing. In that case one can use a scale factor, either during the design or in the final result. We use the figure in the first section as an example:

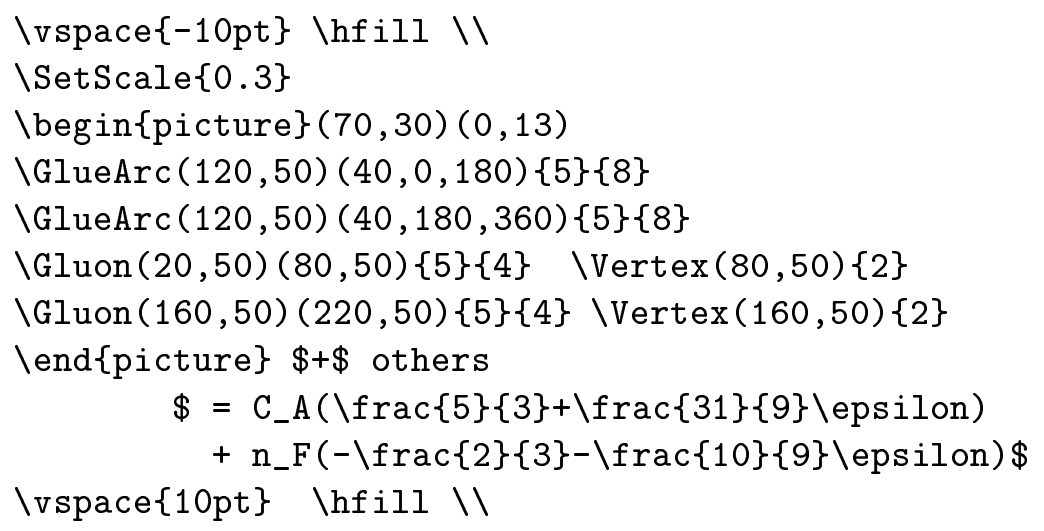

We have lowered the figure by 13 points (the $(0,13)$ in the picture statement) to make it look nice with respect to the equal sign. The result is

$$
\text { לow }
$$

This way it is rather straightforward to make whole pictorial equations. Of course some things are not scale invariant. The appreciation of a figure may be somewhat different when the scale is changed. In the above case one might consider changing the amplitude of the gluons a little bit. Changing this from 5 to 7 and at the same time reducing the number of windings from 4 to 3 for the straight gluons and from 8 to 7 for the gluons in the arcs gives

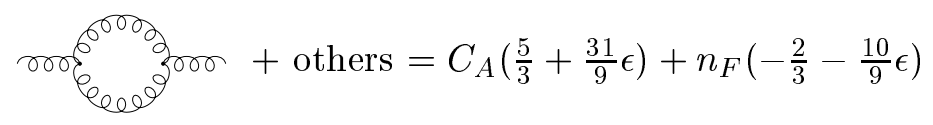

At this scale this may please the eye more.

There is one problem with scaling. Currently it is only possible to have text scale with the rest of a figure when the text has been printed with the PText command. This makes the typesetting more complicated, but the scaling of the $\mathrm{T}_{\mathrm{E}} \mathrm{X}$ pixel fonts would give rather poor results anyway.

\subsection{Photons}

When drawing photons one should take care that the number of wiggles is selected properly. Very often this number should be an integer plus 0.5 . This can be seen in the following example: 


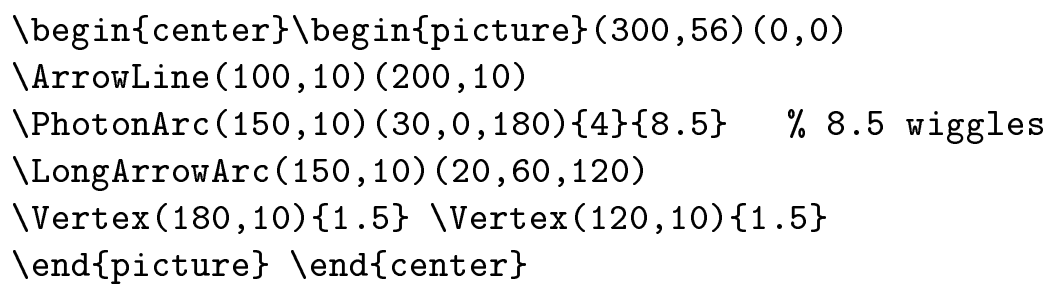

This gives the 'proper' picture as it would usually drawn by hand:

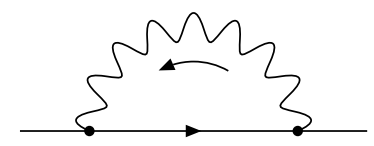

When the number of wiggles is reduced to 8 we obtain:

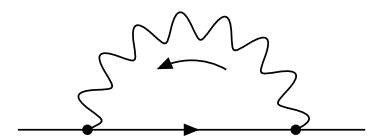

This is not as nice. Somehow the symmetry is violated. One should also take care that the wiggles start in the proper way. If we make the amplitude negative we see that the photons are not 'right' either:

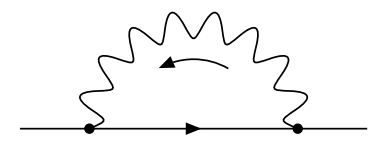

Sometimes these things require some experimenting.

\subsection{Flowcharts}

There are several commands for creating boxes with text in them. This can be a box with either one line of text or with two lines of text. The rest is just a matter of drawing lines and circle segments with arrows. If the text is to scale with the picture one needs to use the postscript fonts. The result of scaling the $\mathrm{T}_{\mathrm{E}} \mathrm{X}$ fonts is usually rather ugly, because these fonts are pixel fonts. Here we present an example. It might describe a system for the automatic computation of cross-sections:

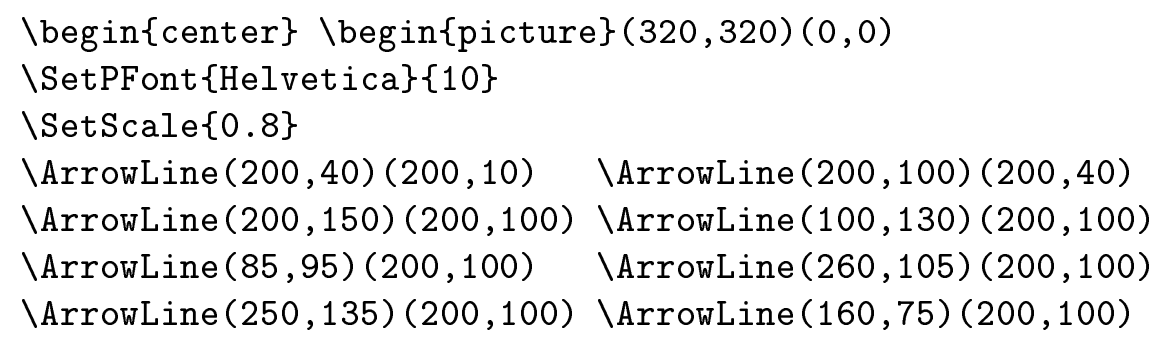




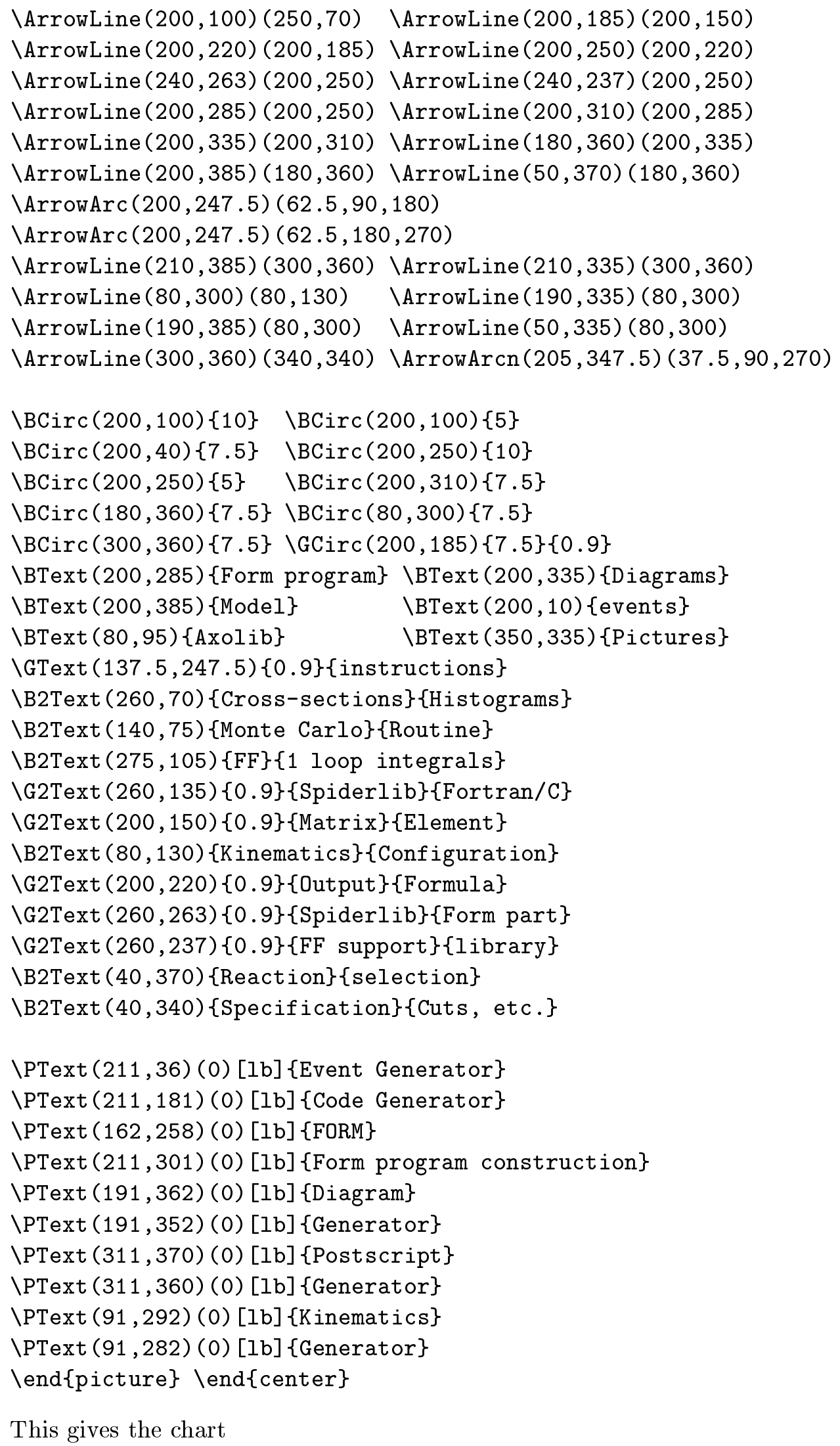




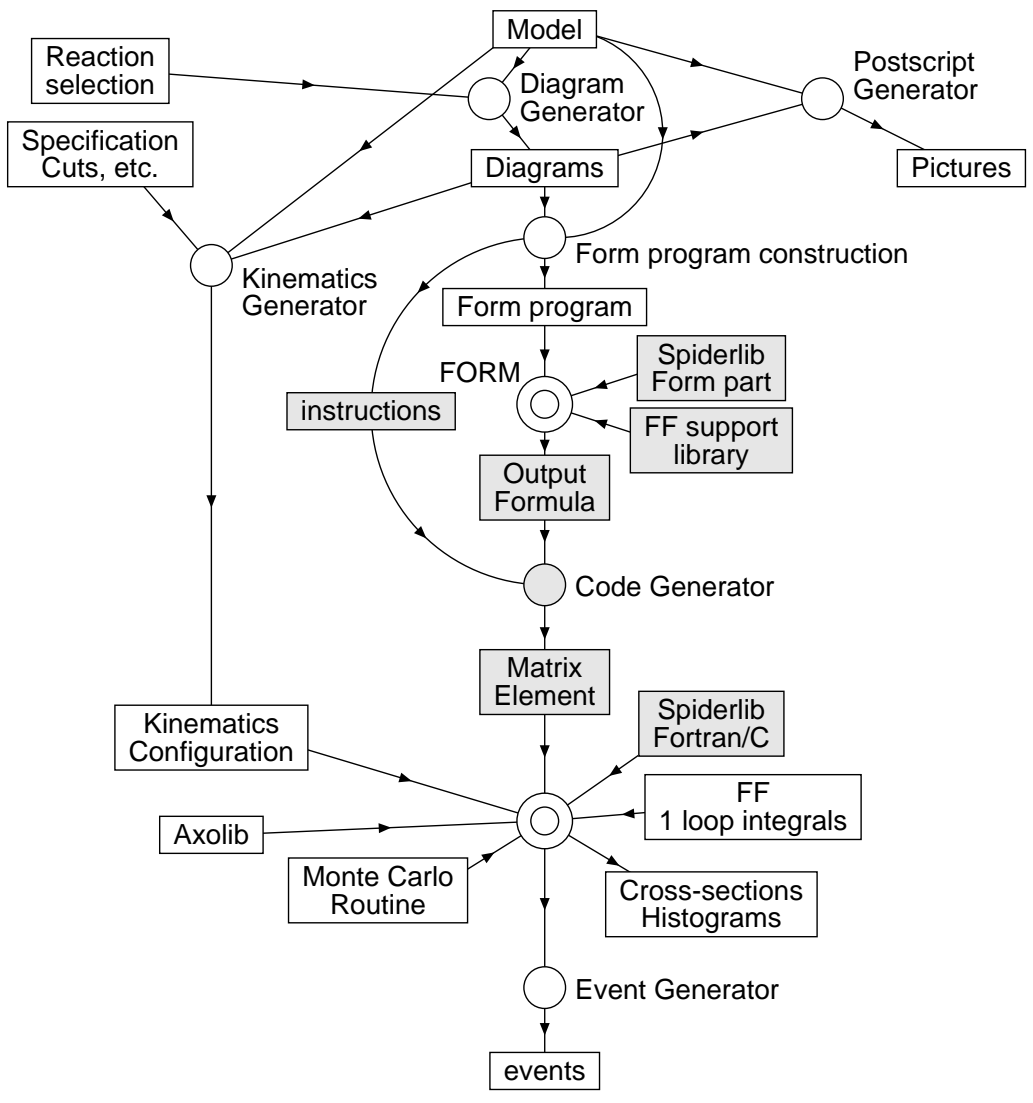

\subsection{Curves and graphs}

Axodraw is equipped with a curve fitting facility that can draw smooth curves through a set of coordinates. Coupled to this is a set of commands to draw the axes that are typically needed for the use of graphs and histograms. An example of a complete picture would be

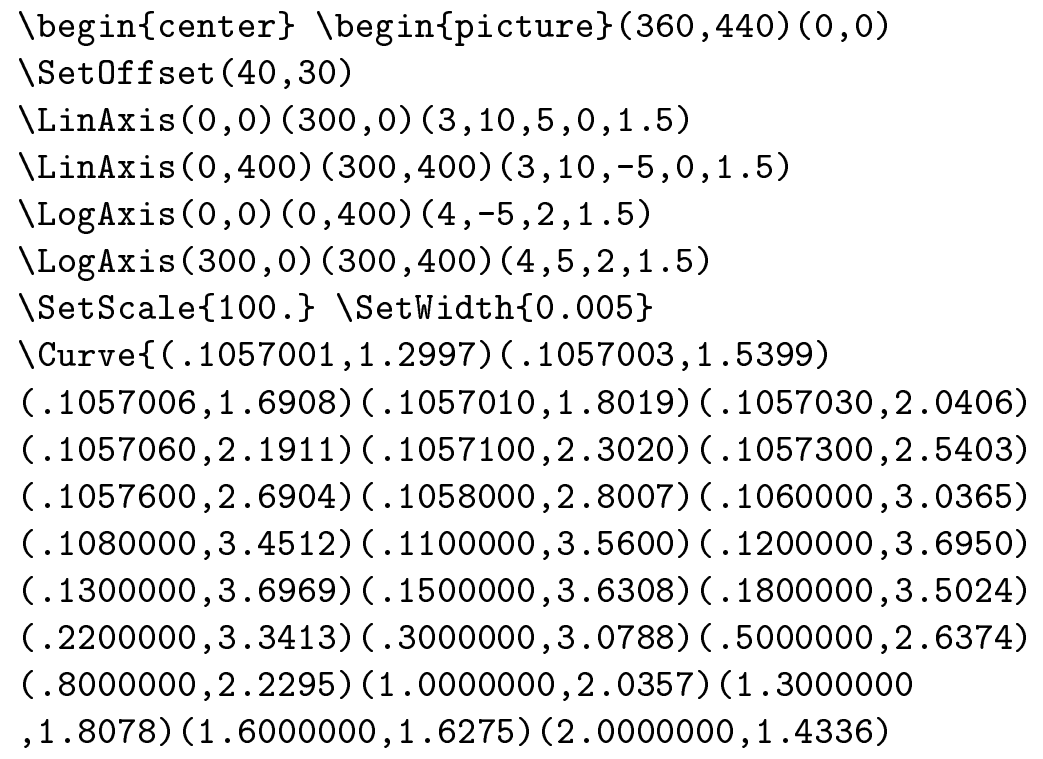




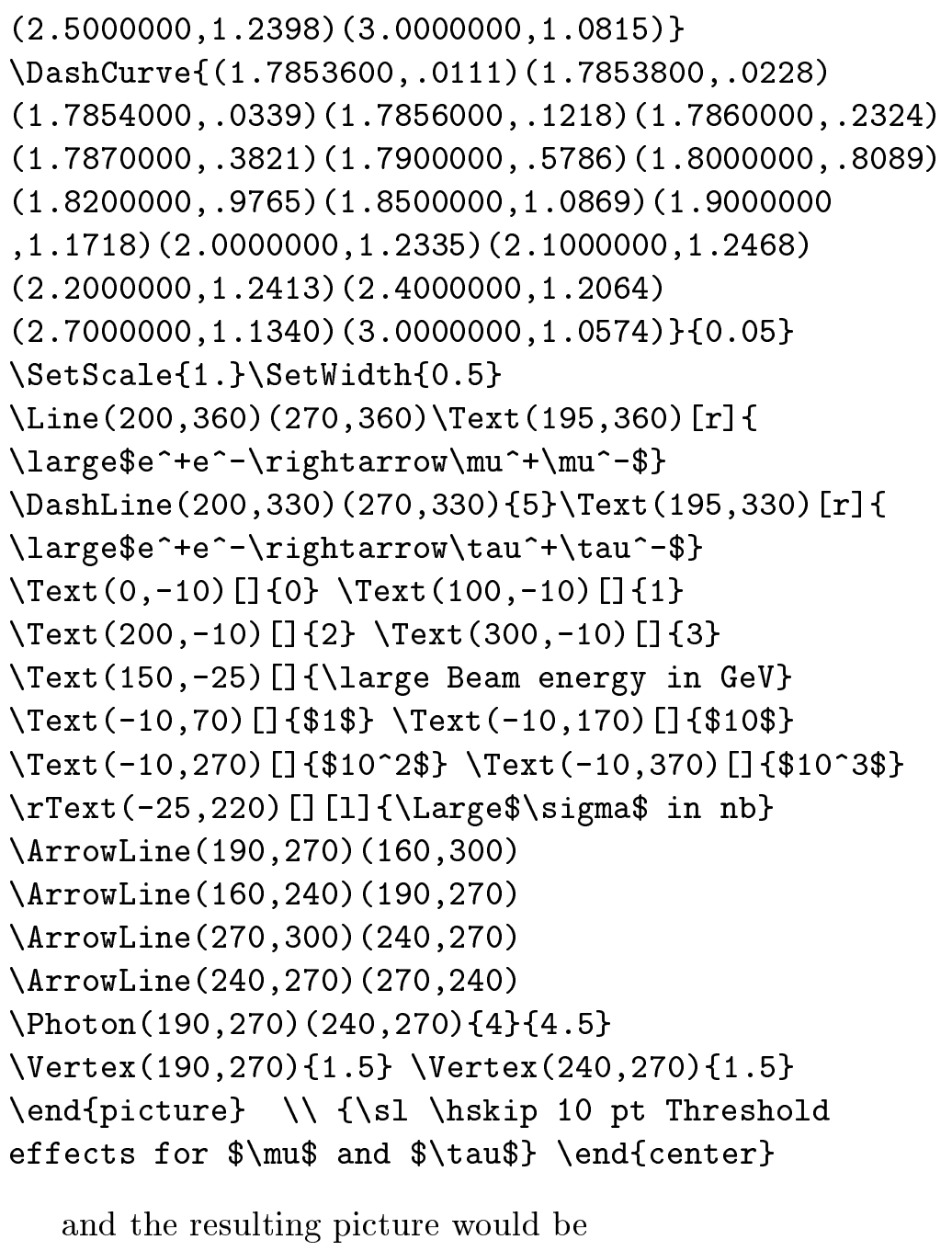




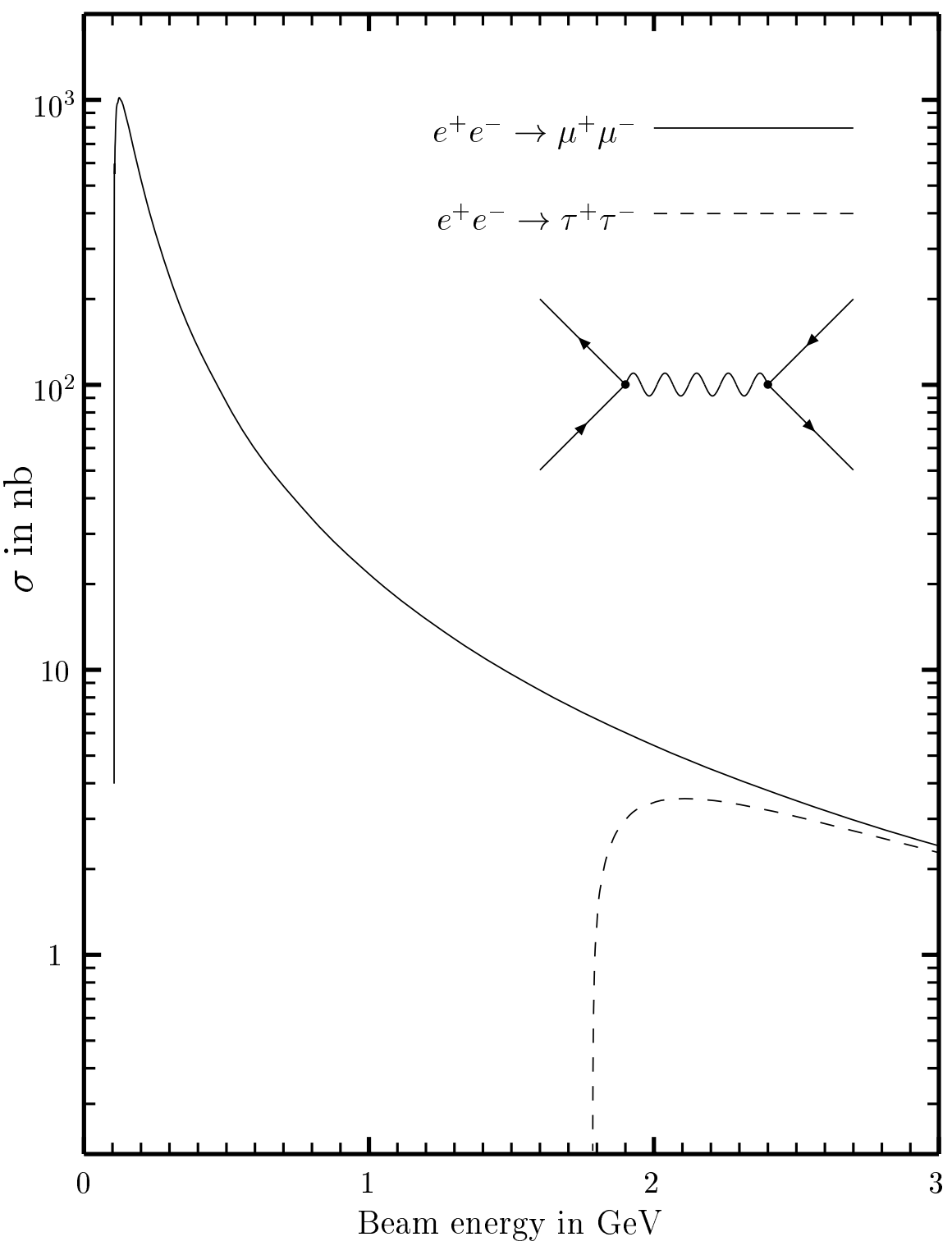

Threshold effects for $\mu$ and $\tau$

Of course one can scale these pictures further, but because the scale factor has been used to enter the data points these should then be adapted too. Note that when the scale is blown up by a factor 100, the linewidth has to be scaled down or disasters will take place.

Finally a playful example:

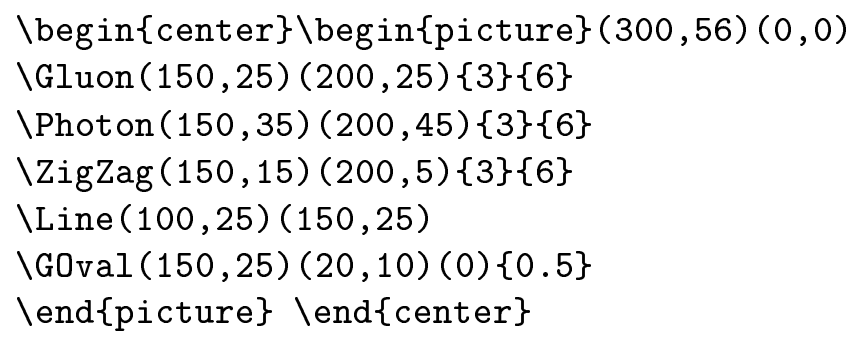


which results in

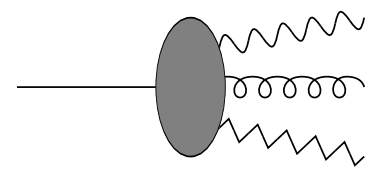

Acknowledgement: The author wishes to thank G.J.van Oldenborgh for help with some of the $\mathrm{T}_{\mathrm{E}} \mathrm{X}$ macros.

Axodraw can be obtained by means of anonymous ftp from ftp.nikhef.nl. It is located in the directory pub/form/axodraw. Commentary and suggestions should be sent to the author at t68@nikhef.nl. 


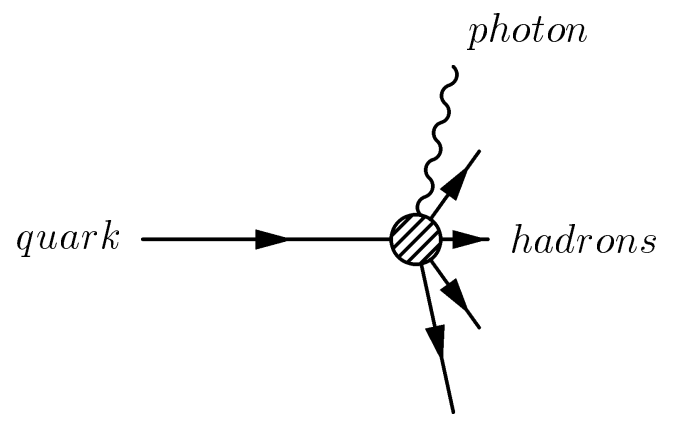

